\title{
Games in Rigged Economies
}

\author{
Luís F. Seoane $\oplus^{1,2,3, *}$ \\ ${ }^{1}$ Departamento de Biología de Sistemas, Centro Nacional de Biotecnología (CSIC), \\ C/ Darwin 3, 28049 Madrid, Spain \\ ${ }^{2}$ Grupo Interdisciplinar de Sistemas Complejos (GISC), Madrid, Spain \\ ${ }^{3}$ Instituto de Física Interdisciplinar y Sistemas Complejos (IFISC, UIB-CSIC), \\ Campus Universitat de les Illes Balears E-07122, Palma de Mallorca, Spain
}

(Received 24 February 2021; revised 25 May 2021; accepted 1 July 2021; published 15 September 2021)

\begin{abstract}
Multiple aspects of an economy can be regulated, tampered with, or left to chance. Economic actors can exploit these degrees of freedom, at a cost, to bend the flow of wealth in their favor. If intervention becomes widespread, microeconomic strategies of different actors can build into emergent macroeconomic effects. How viable is a "rigged" economy? How do growing economic complexity and wealth affect it? We study rigged economies with a toy model. In it, economic degrees of freedom progress from minority to coordination games as intervention increases. Growing economic complexity spontaneously defuses cartels. But excessive complexity leads to large-fluctuations regimes, threatening the system's stability. Simulations suggest that wealth must grow faster than linearly with economic complexity to avoid this regime and keep economies viable in the long run. We discuss a real-case scenario of multiple economic actors coordinated to result in an emergent upset of the stock market.
\end{abstract}

DOI: 10.1103/PhysRevX.11.031058

Subject Areas: Complex Systems,

Interdisciplinary Physics

\section{INTRODUCTION}

At the end of January 2021, a "short squeeze" on Game Stop shares (GME) caused an upset in the stock market [1]. By that time, GME was devalued with a negative prospect; thus, hedge funds invested in "short" options (which make profits if prices drop but are exposed if prices rise). Users of the r/wallstreetbets forum in the Reddit website speculated that GME was artificially undervalued and invested in "long" options (e.g., simply buying and holding stocks, making profits if prices increase). In a short squeeze, traders with short positions must rebuy at higher prices to limit their exposure, which in turn drives stocks further up. Thus, during speculative runs, investors in long options factually pay to increase the likelihood that an economic game favors them and harms others. Short squeezes may result in their orchestrators being charged with market manipulation. This kind of situation is not new-the novelty includes the actors involved (users of a social network), their communication channels, and their access

\footnotetext{
* Corresponding author. brigan@gmail.com

Published by the American Physical Society under the terms of the Creative Commons Attribution 4.0 International license. Further distribution of this work must maintain attribution to the author(s) and the published article's title, journal citation, and DOI.
}

to technology (facilitated by trading apps) that allows a large number of small investors to manipulate the market [2]. As losses for hedge funds mounted up, trading apps limited access to sensitive stocks (while professional brokers could still trade them freely), which prompted class suits amid accusations that the system is "rigged." What dynamics can we expect of rigged marketsespecially as large numbers of small investors move "in sync and en masse" [2] to coalesce in emerging dynamics?

The existence of rigged economic scenarios is amply acknowledged. Most notable examples are noncompetitive markets $[3,4]$, legal or illegal, such as cartels, or natural monopolies [5]. In these examples, all actors usually cooperate to secure similar profits, which entails "handcrafting" some aspects of the economic games in which they engage. In competitive markets, we also find illegal schemes (e.g., insider trading) or innovative, often borderline legal, enterprises to explore unprecedented economic possibilities-e.g., anticipating a broker's moves with faster internet cables [6]. Such out-of-the-box thinking is part of the economy's open-ended nature $[7,8]$, by which the rules of the game are continuously bent, and a sentiment that "the market is rigged" [6] easily emerges. Even if all actors stick to the norms and do not innovate, competitive markets are strongly regulated. Some conditions (e.g., demanding a minimum equity to participate) are designed by governments or international institutions. They might change because of democratic consensus or 
lobbying. If powerful firms bend the rules systematically, regulatory capture happens [9-11], threatening democracy at large $[12,13]$. As transnational markets grow ever more complex and faster, slow public bureaucracies might lag behind and abdicate into nimbler private regulators $[14,15]$.

All these regulations exemplify how, through and through, economies are rigged. Available games are somehow manufactured. Once established, they remain open to manipulations that might (i) impact the costs and rewards of economic games, (ii) cap the information available, or (iii) limit the number of players allowed to partake. These manipulations can be achieved through publicity, bribes, threats, imposing tariffs, etc. More abstractly, we can think of degrees of freedom that can be harnessed in economic systems. Each degree of freedom is a pocket of opportunity that can be exploited (contested or uncontested) at some cost. Envelope theorems assess changes of likely payoffs when a game is altered externally [16-18]. Wolpert and Grana [19] recently built on such theorems to find how much an agent should pay if she (and no other actor involved) could "rig" a game before playing it. The decision boils down to a positive payoff balance with versus without intervention.

In Ref. [19], a single agent is offered control, at a cost, over a single economic degree of freedom. Here, we study what happens when multiple actors are allowed, also at a cost, to manipulate several economic degrees of freedom. Different efforts might align or not, yielding uncertain returns. A single agent's decision to rig one game (as per Ref. [19]) might be of limited consequence in isolation. But effects may be amplified, mitigated, or produce other emergent phenomena when coupled across games and players. We are interested in how microscopic fates scale up to macroeconomic trends (as was the case in the GME short squeeze), so we adopt a systemic perspective. More available degrees of freedom result in more complex economies-intervention possibilities grow combinatorially, and more external variables become relevant if extra degrees of freedom are left unchecked. How do system-wide dynamics of a rigged economy depend on its complexity? How much can such economies grow-openendedly, perhaps? Do they collapse, unable to sustain their participants? How is this affected by the amount of wealth generated and distributed? What is a natural level of intervention depending on these aspects?

We could tackle these questions rigorously through utility functions that discount intervention costs, as in Ref. [19], extended to multiple agents and games; but this quickly becomes untreatable. Instead, inspired by agentbased models and complex adaptive systems [20-25], we model a toy economy that captures essential elements affecting our research questions. We assume a population of agents who engage in $n$ economic games. Each game has a rule that randomly determines its winning strategy. Agents can pay to intervene each game's rule, affecting the winning strategy for all [Fig. 1(a)]. These games constitute available degrees of freedom; thus, $n$ is a proxy for the economy's complexity. An amount of wealth, $B$, is distributed among winners. Since wealth is divided in this way, it might be wise to play the minority strategy, so the earnings are split between less winners. Games in which being in the minority is the winning strategy are called "minority games." Majority games, on the other hand, are situations in which it pays off to play the strategy played by the majority. We will see how the chance to rig economic games shifts between these options as the amount of wealth distributed, $B$, changes. This parameter, $B$, may remind us of a GDP and is a proxy for our economy's size. The model is described in detail in Sec. II. Alternative ways in which economies can be manipulated, not captured by the model, are discussed in Sec. IV.

We write complete payoff matrices for some simple scenarios. Their analysis (Sec. III A) shows that increasing intervention switches isolated degrees of freedom from minority to coordination games. Between both extremes, Nash equilibria are mixed strategies, anticipating dynamic struggles. We explore increasing economy size and complexity with simulations based on agents of bounded rationality and Darwinian dynamics to select successful strategies. We argue (Sec. IV) that our results should not depend critically on the agent's rationality and the choice of Darwinism in the model. We simulate model dynamics for a small, fixed number of degrees of freedom as the economy size grows (Sec. III B). This approach reveals the same progression: from minority, through dynamic, to coordinating regimes. The latter remind us of cartels. Adding degrees of freedom abruptly halts within-game coordination, suggesting an empirical test: Increased economic complexity should dissolve cartels spontaneously. We study our toy economy's viability as its complexity grows large and its size scales appropriately (Sec. III C). Economies whose sizes do not grow fast enough with their complexity fall in a largefluctuations regime that threatens their viability - thus, noncompetitive actions can have negative spillovers as agents and degrees of freedom become coupled en masse. Our toy model allows us to find limit regimes (e.g., within-game coordination, large fluctuations, etc.) that emerge from essential elements potentially common to any rigged economy. We lay out comprehensive maps of such regimes (Sec. III D). Their occurrence is tied to a few abstract parameters. Finally, a brief discussion of the GME short squeeze from the perspective of our results is included (Sec. III E).

\section{METHODS}

\section{A. Model description}

Our toy economy [Fig. 1(a)] consists of a fixed number of games, $n$, and a population of $N(t) \in\left[0, N^{\max }\right]$ agents that changes over time. At each iteration, every agent has to play all games, which admit strategies 0 or 1 . The strategies played by agent $A^{i}$ are collected in an array: $a^{i} \equiv\left[a_{k}^{i}\right.$, $k=1, \ldots, n]$. Besides, a second array $r^{i} \equiv\left[r_{k}^{i}, k=1, \ldots, n\right]$ codifies whether $A^{i}$ attempts to rig game $k\left(r_{k}^{i}=1\right)$ or not $\left(r_{k}^{i}=0\right)$. The combination $\left(a_{k}^{i}, r_{k}^{i}\right)$ constitutes the full 


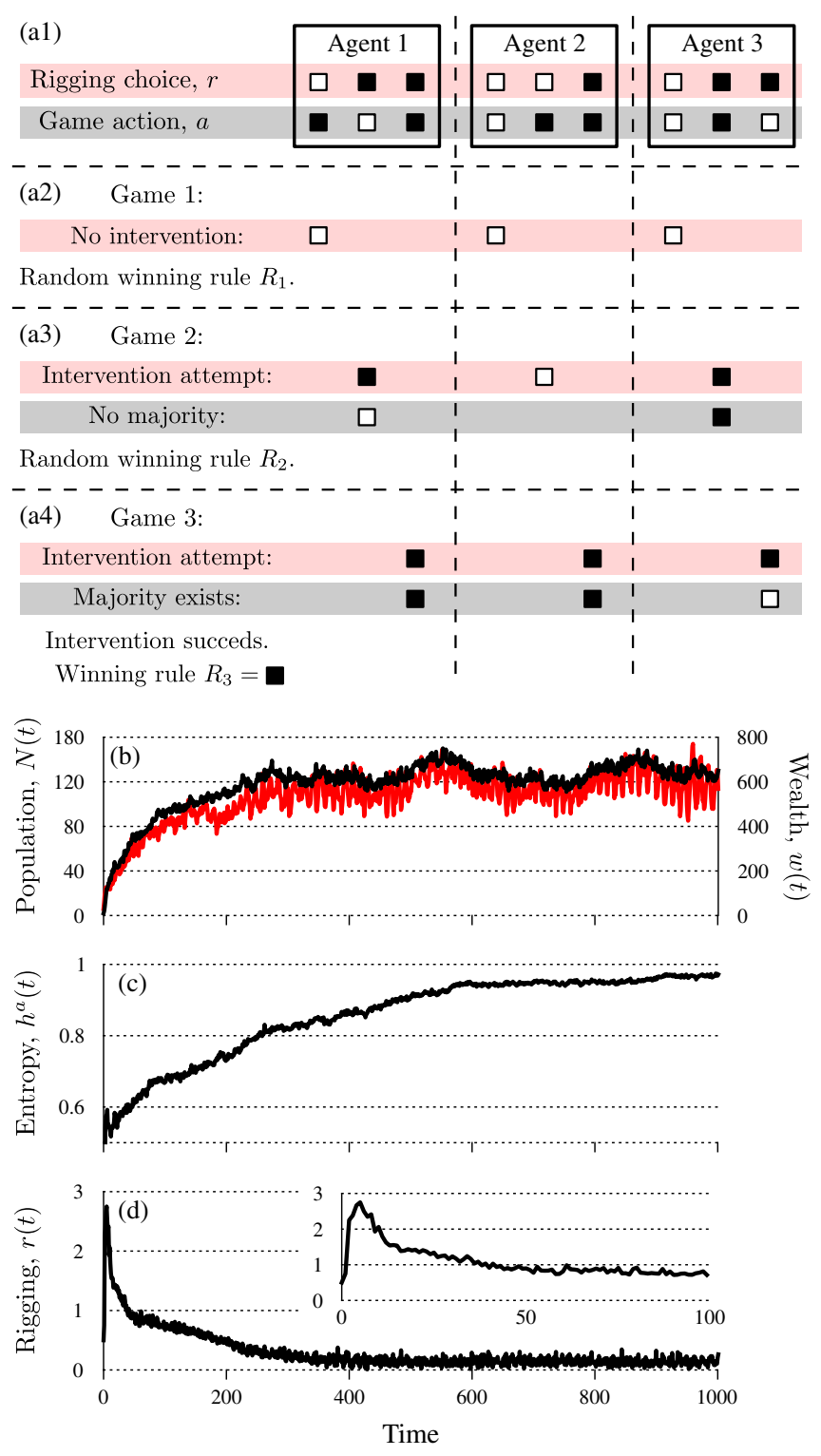

FIG. 1. Rigged economy and its dynamics. (a) Three agents choosing (red shade) whether to rig each game (black boxes) or not (empty boxes), and (gray shade) what to play in each game $\left(a_{k}^{i}=1\right.$ or 0$)$. (b)-(d) Dynamics of model measurements for $C_{C}=10, n=20$, and $B=100$ (thus $b=5$ ). (b) Population (black, left scale) and wealth (red, right scale).

strategy of agent $A^{i}$ towards game $k$. However, to aid the model's discussion, we use the word "strategy" only to name $a_{k}^{i}$.

At each iteration, a rule exists, common to all agents, that determines the winning strategy for each game: $R(t) \equiv$ $\left[R_{k}(t) \in\{0,1\}, k=1, \ldots, n\right]$. If any agents attempt to rig game $k, R_{k}(t)$ takes the most common strategy among those rigging agents [Fig. 1(a4)]:

$$
R_{k}(t)=\underset{\bar{a} \in\{0,1\}}{\operatorname{argmax}}\left(\left\|\left\{A^{i}, a_{k}^{i}=\bar{a}, r_{k}^{i}=1\right\}\right\|\right) .
$$

In the case of a draw (including no intervention), $R_{k}(t)$ is set randomly [Figs. 1(a2)-(a3)]. Each agent pays an amount $C_{R}$ for each intervention attempt-successful or not. If $A^{i}$ has a wealth $w^{i}(t)$ at the beginning of a round, after setting $R(t)$, this becomes

$$
w^{i}\left(t+\Delta t_{\mathrm{rig}}\right)=w^{i}(t)-C_{R} \sum_{k} r_{k}^{i} .
$$

Each round, an amount $b$ is raffled at each game-a total wealth $B=n b$ is potentially distributed. The amount allocated to game $k$ is split between all agents who played the winning strategy, $R_{k}(t)$. After this split in allocation has happened, each agent's wealth reads

$w^{i}\left(t+\Delta t_{\text {play }}\right)=w^{i}\left(t+\Delta t_{\text {rig }}\right)+b \sum_{k} \frac{\delta\left(a_{k}^{i}, R_{k}(t)\right)}{N_{k}^{w}(t)}$,

where $\delta(\cdot, \cdot)$ is Kronecker's delta and $N_{k}^{w}(t)$ is the number of winners of game $k$. If $w^{i}\left(t+\Delta t_{\text {play }}\right)<0$, the $i$ th agent is removed, decreasing the population by 1 .

If $w^{i}\left(t+\Delta t_{\text {play }}\right)>C_{C}, A^{i}$ has a child, and an amount $C_{C}$ is subtracted from $w^{i}$. A new agent is generated, which inherits $a^{i}$ and $r^{i}$. We introduce some variability through mutation: There is an independent probability $p_{\mu}$ that each of the bits in the new agent flips with respect to that of her mother. If a game is not rigged $\left(r_{k}^{i}=0\right)$, there is a chance $p_{\mu}$ that the child will attempt to rig it and vice versa. This mutation chance is resolved independently for each bit in $r_{k}^{i}$ and $a_{k}^{i}$. After this mutation step, both arrays remain fixed throughout the new agent's lifetime. We generate an integer number $j \in\left[1, N^{\max }\right]$ to allocate the new individual. If $j \leq N(t)$, the new agent becomes $A^{j}$. The former agent in that position is removed, its wealth is lost, and the population size remains unchanged. If $j>N(t)$, the new individual is appended at the end of the pool, and the population grows by 1 .

For some context, we might compare our model with the approach in Ref. [19], which also features games that can be intervened at a cost. As noted above, the combination $\left(a^{i}, r^{i}\right)$ constitutes the proper "strategy" of each agent (while we opted to use this word only to refer to $a^{i}$ ), which reveals a duality about rigged economic games, as if two different modules were available. This case is solved in Ref. [19] by playing two sequential stages: In the first one, a single agent plays a game on its own, as she is given the option to change the payoff matrix (this reminds us of our $r^{i}$ ); and in the second one, all agents play the actual game, choosing their strategies according to the information that they have (this maps into our $a^{i}$ ).

The intervening agent in Ref. [19] is free to make any alteration of the payoff matrix, and it is always guaranteed that such intervention is carried out. Hence, the research question becomes as follows: Which modifications are 
satisfactory enough such that it pays off to change the game? In our implementation, any agent is allowed to intervene (as we put the stress on emergent properties), but the interventions allowed are much more limited and probabilistic in nature (as they might not be enforced if enough agents push in the opposite direction). We need these simplifications to get some insight on an issue that quickly becomes complex enough. We hope that both our approach and that in Ref. [19] will be important in furthering this line of research.

\section{B. Measurements on model dynamics}

For each simulation, we set model parameters $\left(C_{R}=1\right.$, $C_{C}=10, p_{\mu}=0.1$, and $N^{\max }=1000 ;$ but variations are explored in the Appendix $\mathrm{C}$ to show the generality of our results). We explore ranges of $n$ and $B$ to address the main questions-i.e., how do rigged economies behave as their complexity and size change?

Model simulations start with a single agent $A^{1}$ with random strategies and no interventions $\left(r_{k}^{1}=0, \forall k\right)$. On average, $A^{1}$ accrues half of the distributed wealth until $w^{1}(t)>C_{C}$. As new descents fill the population, reinforcing or competing strategies unfold. After a rapid initial growth, population size and wealth reach an attractor [Fig. 1(b)]. We assess these attractors numerically. Simulations run for 5000 iterations. We take averages (denoted $\langle\cdot\rangle$ ) of diverse quantities over the last 500 iterations-for example, population size $\langle N\rangle$, for which we also report normalized fluctuations $\sigma(N) /\langle N\rangle$, where $\sigma(\cdot)$ indicates standard deviation. Unless $B$ is very small, 5000 iterations suffice to observe convergence (Figs. 9-11).

To measure the heterogeneity of strategies in the population, we take the fraction $f_{k}(t)$ of agents with $a_{k}=1$ :

$$
f_{k}(t)=\sum_{i=1}^{N(t)} \frac{\delta\left(a_{k}^{i}, 1\right)}{N(t)},
$$

from which we compute the entropy:

$$
\begin{aligned}
h_{k}^{a}(t)= & -\left[f_{k}(t) \log _{2}\left(f_{k}(t)\right)\right. \\
& \left.+\left(1-f_{k}(t)\right) \log _{2}\left(1-f_{k}(t)\right)\right] .
\end{aligned}
$$

If $h_{k}^{a}(t)=0$, all agents are playing the same strategy in game $k$. This quantity is maximal $\left[h_{k}^{a}(t)=1\right]$ if the population is split in half around that game. We also compute a mean entropy across games [Fig. 1(c)]:

$$
h^{a}(t)=\frac{1}{n} \sum_{k=1}^{n} h_{k}^{a}(t)
$$

If $h^{a}(t)=0$, agents play the same strategy in each game but not necessarily the same one across games. If $h^{a}(t)=1$, agents are split in half at each game, but this split is not necessarily consistent across games.
Finally, we introduce the rigging pressure on a game:

$$
r_{k}(t)=\frac{1}{N(t)} \sum_{i=1}^{N(t)} r_{k}^{i}, \quad r_{k}(t) \in[0,1] .
$$

This is the fraction of agents attempting to rig that game. Total rigging pressure across games [Fig. 1(d)],

$$
r(t)=\sum_{k=1}^{n} r_{k}(t), \quad r(t) \in[0, n],
$$

and average rigging pressure per game, $r(t) / n \in[0,1]$, are also reported.

\section{RESULTS}

\section{A. Intervention turns minority into majority games}

Before looking at model dynamics, we can gain some insight from payoff matrices in simple cases. Population size affects these matrices because earnings are split among winners. Also, more agents imply more distinct, possible correlations between strategies and rigging choices. Hence, utility functions rapidly become very complex. In Appendix A, we discuss payoff matrices for a single game and one player (Table II) and for one game and three players (Tables III-V). All matrices show average earnings over time if strategies, rigging choices, and population size are fixed.

Table I presents the payoff matrix for one game with two players. If $C_{R}>b / 2$, rigging the game is prohibitive. Then, the system has the Nash equilibria marked in gray-both agents try to follow opposite strategies $a^{1} \neq a^{2}$. With no intervention, we are dealing with a minority game. For larger populations, it pays even more to be in the minority (Table III). These equilibria disappear if intervention is cheap enough $\left(C_{R}<b / 2\right.$ for one game and two players). Then, it becomes more profitable for one player to rig the game while playing a minority strategy. But it also becomes beneficial, for the other agent, to parasitize the other's effort - turning the winning strategy into a majority. If the game is played sequentially, a dynamic scenario ensues, alternating minority and majority configurations (gray circuits in Table I). As more players are added, the stakes become higher and the situation more complex. Each player that is not attempting to rig the game wants to play the same strategy as the majority among those who are rigging the outcome. Instead, those paying to change the rule of a game prefer that their option is as minority as possible among those who do not pay to intervene. For $n>3$, if all agents are intervening (red frame, Table V), the subgame's Nash equilibrium is a full coordination. This is not a global equilibrium, but large coordinations emerge in our simulations for rising intervention levels (see next section). Note how all agents colluding to rig a game resemble a cartel.

Payoff matrices are equal for all games. If $n$ games are played in isolation (i.e., wealth earned by manipulating a game could not be invested into another), we would 
TABLE I. Payoff matrix of one game with two players. Table entries are labeled by each agent's strategy $a^{1}=0,1$ and rigging choice $r^{1}=0,1$. Each cell displays the average payoff with no death or reproduction for fixed options. Gray cells are Nash equilibria if $C_{R}>b / 2$. Gray circuits indicate possible dynamic situations that emerge for $C_{R}<b / 2$.

\begin{tabular}{|c|c|c|c|c|}
\hline Agent 2 & $a^{1}=0, r^{1}=0$ & $a^{1}=1, r^{1}=0$ & $a^{1}=0, r^{1}=1$ & $a^{1}=1, r^{1}=1$ \\
\hline$a^{2}=0, r^{2}=0$ & $b / 4$ & $b / 2$ & $b / 2 \quad b / 2-C_{R}$ & ${ }_{0} \quad b-C_{R}$ \\
\hline$a^{2}=1, r^{2}=0$ & $b / 2$ & $b / 4$ & $0 \quad b-C_{R}$ & $b / 2 \quad b / 2-C_{R}$ \\
\hline$a^{2}=0, r^{2}=1$ & $b / 2-C_{R}-b / 2$ & $b-C_{R}$ & $b / 2-C_{R}$ & $b / 2-C_{R}$ \\
\hline$a^{2}=1, r^{2}=1$ & $b-C_{R}=0$ & $b / 2-C_{R} \quad b / 2$ & $b / 2-C_{R}$ & $b / 2-C_{R}$ \\
\hline
\end{tabular}

observe the same transition to within-game coordination for each degree of freedom as intervention takes hold. What happens when we lift such compartmentalization?

\section{B. Fixed complexity and growing wealth}

We now study model dynamics and stability for a fixed number of games and varying economy size. A discussion of the rich behavior uncovered is given in Appendix B.

Figure 2(a) shows $\langle N\rangle$ for $n=2$ games. Circles over the plots indicate values of $B$ for which a stretch of the dynamics is plotted in Fig. 15 in Supplemental Material [26]. Generally, $\langle N\rangle$ increases with the economy size-i.e., as more money becomes available to sustain more agents or to invest into rigging more games. Indeed, the rigging pressure per game [Fig. 2(b)] grows, more or less, monotonously. Note that $\langle N\rangle$ is not so parsimonious. For roughly $B<750$, it grows steadily. At $B \simeq 750$, it jumps swiftly, then remains similar but slightly declining up until $B \simeq 1600$, when it undergoes another abrupt increase.

These population boosts seem to be associated with varying coordination. Figure 2(c) shows that the strategy entropy $\left\langle h^{a}\right\rangle$ drops sharply before the first boost (shaded area). Before that drop, resources are scarce and rigging the economy is difficult. Either strategy is equally likely to win, so agents playing either option are equally abundant [Fig. 15(a) in Supplemental Material [26] ]. As B grows, more resources become available to rig the games. Either 1 or 0 becomes the winning strategy over longer time stretches, resulting in temporary selective preferences for one strategy over the other, and oscillatory dynamics ensue [Figs. 15(b) and 15(c) in Supplemental Material [26] ]. As $\left\langle h^{a}\right\rangle$ falls definitely, agents coordinate their strategies [Figs. 15(d) and 15(e) in Supplemental Material [26]]. These shifts between dynamics happen simultaneously in all games - as if, so far, payoff matrices were essentially independent for each degree of freedom. By $B \simeq 1000$, we have exhausted all within-game regimes uncovered in payoff matrices: from minority games to a mostly majority game and, in between, through ill-defined Nash equilibria that appear as a dynamic struggle in the agent-based model, with winning strategies changing (erratically at first, cyclically at last) as games are played sequentially. The final population boost at $B \simeq 1600$ must entail emerging correlations across games-e.g., clustering agents that play the minority-vs-majority strategies in both games.

Figures 9 and 10 compare $\langle N\rangle,\langle r\rangle / n$, and $\left\langle h^{a}\right\rangle$ as the economy size grows for different, fixed $n$. With more games, more discrete jumps in $\langle N\rangle$ appear. These jumps arise, potentially, from the combinatorially growing coordination possibilities across games. They happen after the oscillatory phases [Figs. 8 and Figs. 16(c)-16(e) in Supplemental Material [26] for $n=3$ ], which again suggests that withingame coordination happens first, simultaneously for all
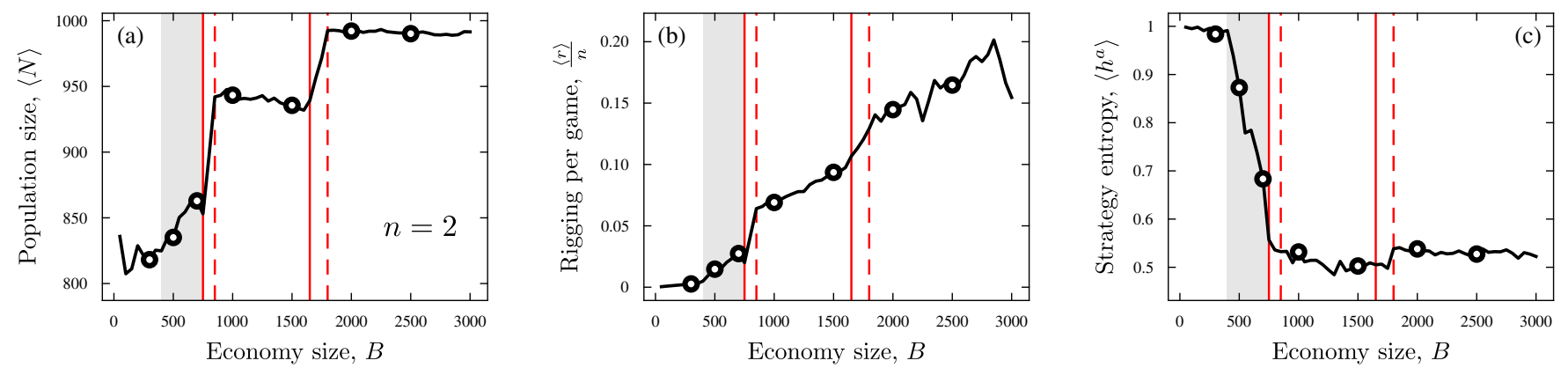

FIG. 2. Model behavior for $n=2$ games and growing wealth, $B$. (a) $\langle N\rangle$, (b) $\langle r\rangle / n$, and (c) $\left\langle h^{a}\right\rangle$. Open circles indicate $B$ values for which we plot sample dynamics in Fig. 10 in Supplemental Material [26]. Red vertical lines loosely indicate discrete jumps in $\langle N\rangle$. 

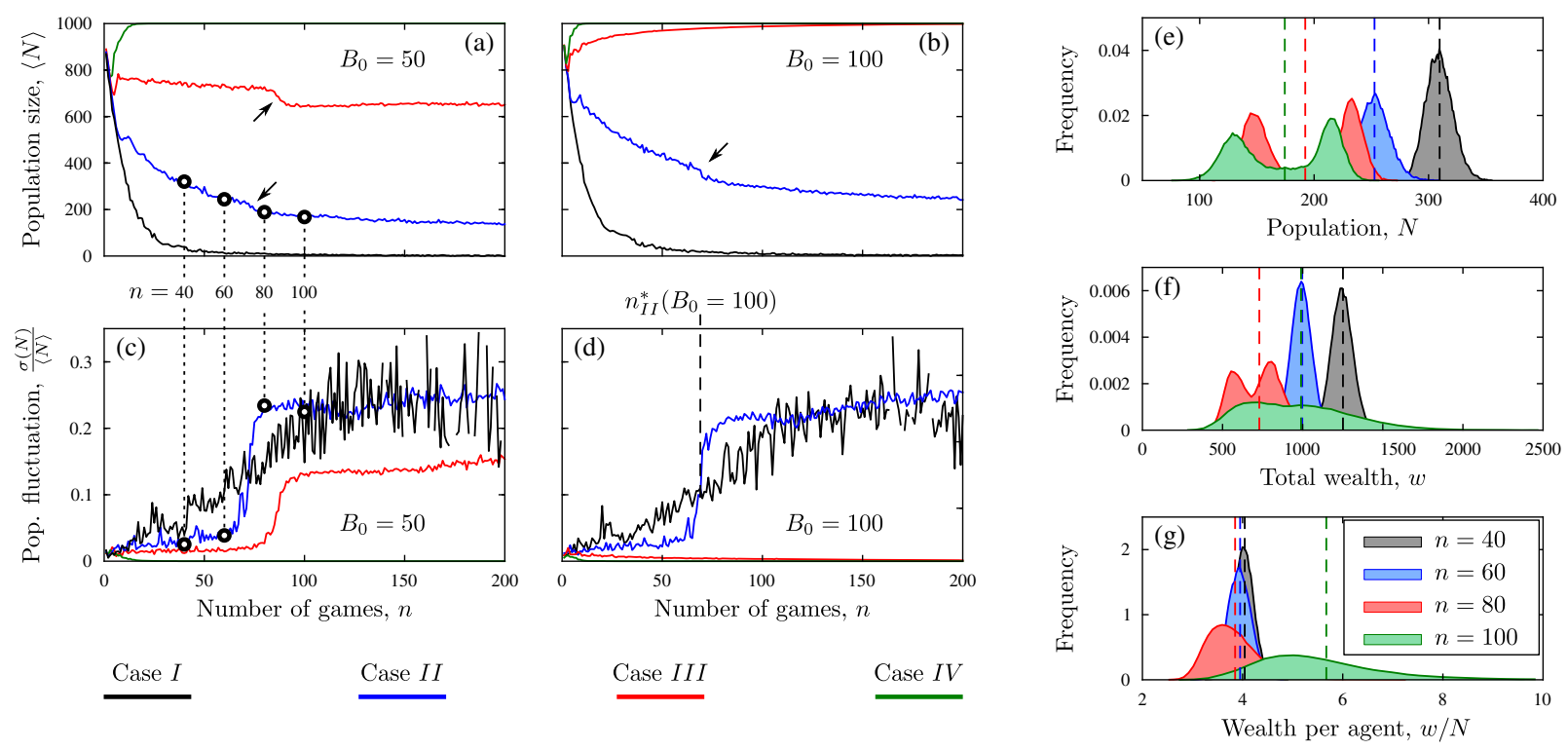

FIG. 3. Population size and fluctuations for growing $n$ and $B \equiv B\left(B_{0}, n\right)$. Panels (a) and (b) show $\langle N\rangle$, and (c) and (d) show $\sigma(N) /\langle N\rangle$. (a,c) $B_{0}=50 ;(\mathrm{b}, \mathrm{d}) B_{0}=100$. Arrows mark the onset of large fluctuations in $\langle N\rangle$. Dotted, vertical lines and circles in panels (a) and (c) indicate $n$ values examined in panels (e)-(g). (e) Probability density functions of population size, $N$; (f) total wealth $w$; and (g) wealth per capita $w / n$.

games; then, degrees of freedom start coupling with each other. Some regimes have similar $\langle N\rangle$ for different $n$ [horizontal dashed lines, Fig. 9(a)], suggesting that they are effectively similar-i.e., that some economic degrees of freedom in the case with larger $n$ might have collapsed into a single one. Population boosts succeed each other more rapidly for larger $n$, approaching a continuous buildup instead of discrete jumps [Fig. 10(a)]. This continuous buildup is not accompanied by a decrease in $\left\langle h^{a}\right\rangle$, which only drops once because of within-game coordination. The $\left\langle h^{a}\right\rangle$ plateau is higher for larger $n$ [Fig. 10(c)], indicating that across-game correlations weaken or interrupt within-game coordination. Above, we compared such coordination to cartels: Games are consensually rigged to favor most actors. The higher $\left\langle h^{a}\right\rangle$ plateaus for larger $n$ suggest that increasing economic complexity prevents the formation of such consensus, defusing cartels, even with rising intervention levels. Exploration of a variant of the model (Appendix C) suggests that, for this strategy to work properly, the degrees of freedom added to the system must be of similar importance to the one whose consensus we wish to prevent-otherwise, the approach can backfire. All of these conclusions of the model are testable.

\section{Growing wealth and economic complexity}

We now change the number of games as wealth scales as $B=B\left(B_{0}, n\right)$. The constant $B_{0}$ is a normalizing factor to facilitate comparisons. We explore four cases:

(I) A fixed wealth $B_{I}=B_{0}$ is split evenly between all games: $b_{I}=B_{0} / n$. Returns per game drop as the economy becomes more complex.
(II) Each game distributes a fixed amount $b_{I I}=B_{0}$, such that total wealth grows linearly $B_{I I}=B_{0} \cdot n$. Returns per game remain constant against growing complexity.

(III) Each degree of freedom revalues previously existing games logarithmically: $b_{I I I}=B_{0}(\log (n)+1)$. Total wealth grows as $B_{I I I} \sim B_{0} \cdot n(\log (n)+1)$.

(IV) Each degree of freedom revalues previously existing games linearly: $b_{I V}=B_{0} \cdot n$. Total wealth grows quadratically: $B_{I V}=B_{0} \cdot n^{2}$.

Figures 3(a) and 3(b) show $\langle N\rangle$ for each scenario. Extreme cases I (black curves) and IV (green) are relatively uninteresting: Stable population size declines quickly for I. As the economic complexity grows and returns per game drop, more intervention is needed to secure the same earnings. Such rigged economies collapse if they become too complex. For IV, wealth grows so quickly with $n$ that, promptly, population saturates.

Intermediate cases II (blue curves) and III (red) are more interesting. With $B_{0}=50$ [Fig. 3(a)] and $B_{0}=100$ [Fig. 3(b)], $\langle N\rangle$ declines slowly for II. Thus, in general, a rigged economy's wealth must grow faster than linearly with its complexity to remain viable. In case III, $\langle N\rangle$ saturates for $B_{0}=100$ but not for $B_{0}=50$, for which population seems stagnant.

For case II, and case III with $B_{0}=50$, fluctuations in population size reveal the existence of thresholds, $n_{I I / I I I}^{*}\left(B_{0}\right)$, at which system dynamics change abruptly [Figs. 3(c) and 3(d)]. This change affects $\langle N\rangle$ marginally [arrows in Figs. 3(a) and 3(b)], but the increase in $\sigma(N) /\langle N\rangle$ is always salient. For $n<n^{*}$, fluctuations are small $(<5 \%)$. For $n>n^{*}\left(B_{0}\right)$, large fluctuations (about 

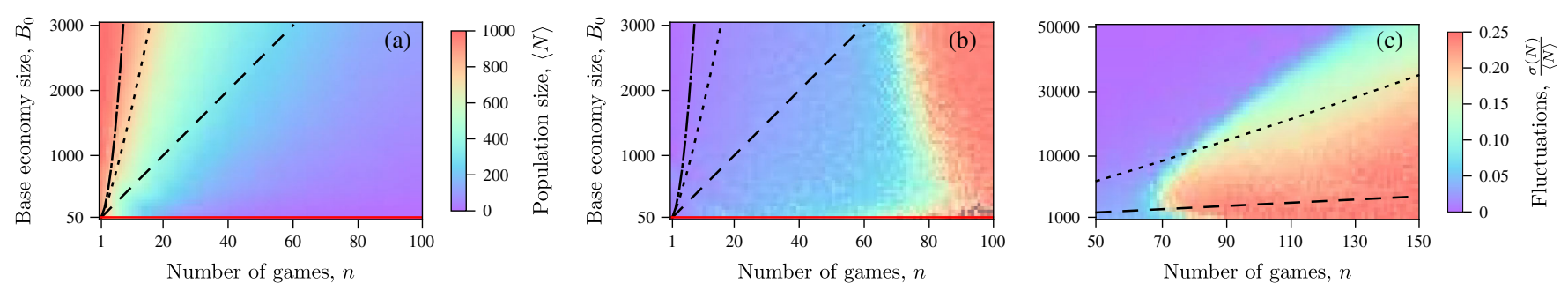

FIG. 4. Comprehensive maps of $\langle N\rangle$ and $\sigma(N) /\langle N\rangle$. Maps result from simulating case I (i.e., $B_{I}=B_{0}, b_{I}=B_{0} / n$ ) for ranges of economic complexity $n$ and distributed wealth, $B_{0}$. Black curves represent trajectories $B=B\left(B_{0}=50, n\right)$ for cases II, III, and IV (dashed, dotted, and dot-dashed, respectively). Horizontal red lines at the bottom of maps (a) and (b) represent case I with $B_{0}=50$. Panel (a) shows $\langle N\rangle$, and panels (b) and (c) show $\sigma(N) /\langle N\rangle$ over two ranges of $n$ and $B_{0}$.

$25 \%$ for case II and about $15 \%$ for case III) set in. There is an absorbing state at $N(t)=0$; thus, fluctuations of $15 \%-$ $25 \%$ of the system size can compromise its viability.

We explore the transition to large fluctuations by simulating case II below $(n=40,60)$ and above $(n=80,100)$ their onset at $n^{*}$. We ran the model for 5000 iterations and discarded the first 1000. Figure 3(e) shows the probability of finding the system with population $N$. Below $n^{*}$, we see a neat Gaußian; above, the distribution presents two balanced modes. Transition between both modes contribute to the large fluctuations. We also plot total wealth [Fig. 3(f)] and wealth per agent [Fig. $3(\mathrm{~g})]$. Their averages fall first, and grow, eventually, as $n$ increases. Below $n^{*}$, clear Gaußians appear again. Above, we observe broad tails, indicating large inequality. Despite risking collapse, the average agent can be wealthier in the large-fluctuations regime.

\section{Charting rigged economies}

We run simulations of case I $\left(B_{I} \equiv B_{0}, b_{I} \equiv B_{0} / n\right)$ for ranges of economic complexity $n$ and distributed wealth $B_{0}$. This process renders maps (Fig. 4) where the four scalings above can be read as curved sections. Trivially, case I traces a horizontal line (solid, red; bottom of each map). Case II traces a line with slope $B_{0}$ (dashed black lines). Cases III (dotted black) and IV (dash-dotted black) trace curves growing faster than linearly. By reading $\langle N\rangle$ [Fig. 4(a)] or $\sigma(N) /\langle N\rangle$ [Figs. 4(b) and (c)] along such curves, we get the plots from Figs. 3(a)-3(d). Results for fixed $n$ and growing $B$ from Fig. 2 result from vertical cuts of the map. Other possible progressions $B=B(n)$ can be charted similarly.

The large-fluctuations regime is a salient feature [Figs. 4(b) and 4(c)] expanding upwards and rightwards (perhaps unboundedly) over a broad range of $(n, B)$ values. Its contour constrains dependencies, $B=B(n)$, that could avoid this regime. Its upper bound seems to grow faster than $n \cdot \log (n)$, suggesting that case III with $B_{0}=50$ will not escape large fluctuations despite sustained growth.

Figure 11 shows maps for $\left\langle h^{a}\right\rangle$ and $\langle r\rangle / n$. The dent of low $\left\langle h^{a}\right\rangle$ due to within-game coordination in simple yet wealthy setups is notable [Fig. 11(a)]. We argue that such cartel-like cases could be defused by increasing complexity. But this map shows that, if $n$ grows too much without increasing $B,\left\langle h^{a}\right\rangle$ drops gradually-consensus strategies build up again. It is intuitive that rigging pressure per game $(\langle r\rangle / n)$ grows alongside $B$ [Fig. 11(b)] since more available resources can be dedicated to rigging the games. Less intuitively, our map shows $\langle r\rangle / n$ growing with $n$ as well, even if returns per game diminish. We speculate that, for low $n$, different agents meddling are likely to collideresulting in uncertain returns and less incentive to intervene. With larger $n$, different agents can focus on rigging different degrees of freedom, lowering the chance of mutual frustration-thus resulting in higher rigging pressure per game as more become available.

\section{E. A real case unfolds-Game Stop short squeeze}

Our model is thought of as a conceptual tool to chart emerging phenomena in rigged economies. It is not designed to predict specific trends. However, we can discuss real cases, outlining similarities, differences, and expected outcomes based on model parameters. Here, we look briefly and qualitatively at the GME short squeeze from early 2021 (Fig. 5). In Appendix D, we discuss a series of markets from the perspective of "rigged" games. In that speculative exercise, we try to find features that might be useful to model from this same viewpoint.

In late 2020, prospects for GME were negative, and numerous hedge funds had adopted short positions. To do this, they "borrowed" GME stock from shareholders and sold them in the stock market. Since GME was expected to lose value, they would later rebuy shares at a lower price before returning them to the original borrowers, allowing traders to profit from a drop in price. Within our toy model, such devaluation could be the outcome $R_{\mathrm{GME}}=0$ in this economic game. If GME had actually devalued, traders that had played the strategy $a_{\mathrm{GME}}=0$ (i.e., "short" speculation) would have reaped and shared the corresponding profits. But throughout January 2021, users of the forum r/wallstreetbets on the website Reddit invested on GME en masse [Fig. 5(b)]. Within our model, simply holding shares (instead of selling and rebuying) is equivalent to playing strategy $a_{\mathrm{GME}}=1$, which pays off if the prices increase in 

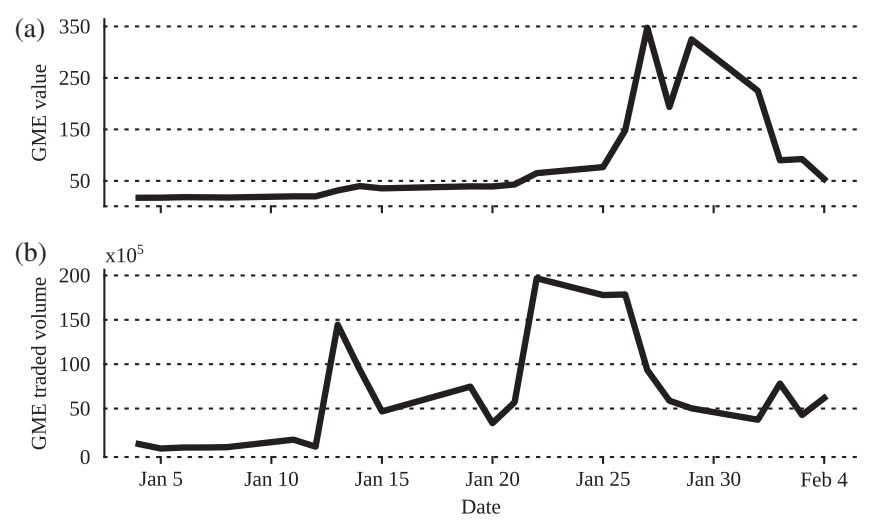

FIG. 5. GME short squeeze. (a) GME value at closing. (b) Daily traded volume of GME stock. Users of r/wallstreetbets built the foundation for the short squeeze during the month of January 2021 [1], as noted by the increased traded volume up until January 27. The share price rose eventually over $1500 \%$ on Jan 27 (with respect to the value two weeks earlier). Afterwards, trade volume returned to a low level as small investors played their "hold" strategy. Despite their efforts, the bubble seemed to be deflating by February 4.

the long term $\left(R_{\mathrm{GME}}=1\right)$. Furthermore, given the speculation following discussions in r/wallstreetbets, each share purchased raised GME prices. Thus, buying additional GME stock is equivalent to exerting a rigging pressure $\left(r_{\mathrm{GME}}=1\right)$ in this economic game to favor the outcome $a_{\mathrm{GME}}=1$. As traders with short positions had to return their borrowed shares, they needed to rebuy-now at a much higher price-which would drive the prices further up, thus exerting further rigging pressure. Differently from our model, agents playing $a_{\mathrm{GME}}=1$ forced agents playing $a_{\mathrm{GME}}=0$ to manipulate $R_{\mathrm{GME}}$ in a direction opposed to their own strategy.

But there are additional ways to rig this economic game. The pressure towards higher GME values came from thousands of small investors trading through online apps. Blocking access to app users would stop the trend-as effectively happened. Within our model, this would be a rigging pressure towards $R_{\mathrm{GME}}=0$. The cost of this strategy is potentially higher since trading apps that blocked GME later faced class suits for alleged market manipulation, as well as threats of political investigations. The affected entities dismissed the charges, arguing that GME trading was halted because of technical reasons (e.g., to protect app users from high volatility or to provide additional collateral demanded by the unexpected price surges). The case was still unfolding as of the writing of this paper.

In parallel with GME, AMC Entertainment Holdings (AMC) and the technology firm BlackBerry (BB) were the subject of similar speculation in online forums, resulting in short squeezes of much smaller magnitude. Small investors diverting their efforts to more than one stock remind us of increased degrees of freedom in our toy model, which, as we saw, promptly degrades established consensus [Figs. 9(c) and 10(c)]. Some apps halted trading of AMC and BB shares as well. Attending to our model, a more effective (and potentially cheaper) strategy to defuse the consensus could have been to facilitate (even promote) access to these and other stocks. Part of the effort by small investors was to "hold" their positions in GME; thus, their ability to coordinate across several games might have been limited.

This real case presents important departures from our model, which suggest future lines of research. In our model, all agents pay $C_{R}$ to attempt to rig each game. In reality, the effort to drive prices up can vary, as investors choose how many shares to buy. Our model can be modified to allow agents to decide how much they want to invest in each intervention, with their influence being weighted accordingly, perhaps reproducing a heterogeneity of strategies observed in actual markets. On the other hand, professional traders and stock markets have the upper hand in trying less orthodox interventions (such as halting trade), the cost of which is difficult to assess. Opposed to our model, profits also depend on the invested effort. In our model, agents must play all games available, while GME investors could decide whether to enter additional games (e.g., AMC) or not. Modeling these features might uncover additional phenomenology for games in rigged economies, but the regimes described in this paper stand as limit cases and a sample of what can be expected. In Appendix C, we illustrate how the relevant regimes are found in one of several model variations in which we tried to check that the uncovered phenomenology is robust.

\section{DISCUSSION}

It is difficult to pinpoint what an "unrigged" economy is. We model economies as containing degrees of freedom that can be controlled at a cost by its actors. Unchecked degrees favor economic agents at random. An economy with more "riggable" facets is more complex. We studied the dynamics, stability, and viability of a rigged economy toy model as its complexity and total wealth changed.

Simple scenarios allow a study of equilibria in payoff matrices. We find that individual degrees of freedom turn from minority into majority games (through a dynamical phase) as intervention increases. Agent-based simulations confirm these regimes. They also show new behaviors as synergies develop between degrees of freedom. These new behaviors (difficult to capture with payoff matrices) halt within-game coordination. Within-game coordination in simple yet wealthy markets resembles cartels: Most economic actors with decision power bend the rules homogeneously in their favor. Our results suggest that this consensus is spontaneously defused if the system becomes complex enough, which can be empirically tested.

We study our toy economies as their complexity increases and the wealth they distribute remains constant 
(case I), grows linearly (case II), or grows faster than linearly (cases III and IV) with the number of economic degrees of freedom. In general, wealth should grow faster than linearly. Against rising complexity, stagnant or slowly growing wealth only sustains a decreasing ensemble of actors sharing ever more meager resources. An unlucky fluctuation can kill them off. This becomes more pressing as our model predicts that large fluctuations build up abruptly above a complexity threshold. These large fluctuations remind us of chaotic regimes in the El Farol and similar problems [20-23]. In them, agents with sufficient rationality anticipate a market, but their own success makes the market unpredictable. In our model, above a complexity threshold, noncompetitive intervention choices become intertwined across games. Birth and death of agents ripple system wide, making successful strategies hard to track. Even though agents are exploring noncompetitive strategies, large fluctuations (15\%-20\% population size) ensue, compromising the system's viability-thus, noncompetitive actions can result in negative spillover by sheer market complexity, which is another testable conclusion.

Behavioral economics offers a prominent chance to test our findings. We see stable states with rising rigging pressure as expected returns grow. This result is consistent with empirical data on experiments that allow cheating: People with different profiles exist (including those who are hardly dishonest), but in such experiments, cheating eventually ensues for large enough rewards [27], especially after removing the concern of being caught [28]. Further experiments reveal that cheating is more likely done as a partnership [29]. This resounds with our model's "cartels" in simple yet wealthy economies. Such simple experiments are perfect to test our predictions for growing complexity: Does coordination fall apart swiftly? Does rigging pressure grow with complexity in the long run? More ambitiously, we could emulate recent implementations of Prisoner's dilemmas and other simple games [30-39].

In this work, we did not aim at specific realism but at capturing elements that we find essential about rigged economies. From these elements, we derive qualitative regimes and wealth-complexity scalings that keep our toy economies viable. Exploring lesser model parameters (Appendix C) and several variations of the original model (one of which is reported in Appendix C as well), the same phenomenology is consistently featured. This suggests that we are unveiling general results of rigged economies. However, we made important simplifications to keep our model tractable. All agents participate in all games, while real economic actors might walk out or be banned from a specific market. We model all degrees of freedom with a similar game. Real manipulations might treat agents with the same strategy differently. Some real games pay off only if you are the first to intervene; in others, the reward grows (even nonlinearly) with the investment on rigging the outcome. Exploring these and other alternatives is easy and might uncover new systemic regimes. Our results constitute solid limit behaviors that should be recovered under appropriate circumstances.

In our model, wealth is generated externally-the economic games merely distribute it. An important variation should create wealth organically, depending on population size, strategies explored, and degrees of freedom available. In such organically growing economies, additional available games would develop and be sustained at a cost. Rigged economies might then correct themselves by losing complexity if necessary. Similar feedback can poise complex systems near critical regimes [40-47], which proved relevant to rationalize some phenomenology in economics $[23,25,48]$ - at criticality, we observe fat tails in wealth distributions or dynamic turnover of complex markets.

An important design choice is the Darwinian dynamics, which propagates successful strategies. We could have modeled boundedly rational agents that learn-similarly spreading successful behaviors. A key parameter would then be the learning rate, instead of our replication cost $C_{C}$. Similar models show that certain regimes depend tangentially on the cognitive mechanism [20-23]. Different implementations might have a different key parameter that changes the onset of large-fluctuations regimes (as $C_{C}$ does, Fig. 12). When unpredictability is intrinsic to the phenomena studied, unboundedly rational agents cannot perform better either. Our results suggest that rigged economies might be intrinsically uncomputable in certain limits.

Our work is designed in economic terms, but it has an obvious political reading - e.g., construction and control of power structures. More pragmatically, in our model, wealth redistribution is achieved through low rigging pressures. Empirical measurements of redistribution might help us map real economies into our framework (similarly to how real economies were mapped into other toy models $[24,25])$. In general, applying our model to politics could help us assess the evolutionary stability of fair governance [15]. In ecosystems that bring together wealth, people, and economic games, all subjected to Darwinism, what lasting structures emerge, do fair rules survive, and under which circumstances does unfairness prevail?

Code needed to simulate the model is provided through a public repository [49].

\section{ACKNOWLEDGMENTS}

The author wishes to thank Roberto Enríquez (Bob Pop) for his deep insights in socioeconomic systems, which prompted this work. The author also wishes to thank Juan Fernández Gracia, Víctor Eguíluz, Carlos Melián, and other Institute for Interdisciplinary Physics and Complex Systems (IFISC) Carles García O'Dowd, Víctor Notivol, Paolo Barucca, David Wolpert, Justin Grana, and, especially, Federico Curci for indispensable feedback about economic systems. This work has been funded by IFISC as 
part of the María de Maeztu Program for Units of Excellence in R\&D (MDM-2017-0711), and by the Spanish National Research Council (CSIC) and the Spanish Department for Science and Innovation (MICINN) through a Juan de la Cierva Fellowship (No. IJC2018-036694-I).

\section{APPENDIX A: PAYOFF MATRICES FOR SIMPLE CASES}

Let us take one game $(n=1)$, a fixed population of one player $N(t)=1$, and no life and death dynamics (i.e., even if the agent accumulates wealth, she does not have descendants, so she never pays $C_{C}$; she is not removed from the population either if her wealth becomes negative). Table II shows the average payoff for such an agent with fixed behavior (i.e., fixed strategy $a_{1}^{1}$ and rigging choice $\left.r_{1}^{1}\right)$. With no intervention $\left(r_{1}^{1}=0\right), R(t)$ is set randomly at each iteration, and the expected payoff per round is $b / 2$. If the agent attempts to rig the game $\left(r_{1}^{1}=1\right)$, she always succeeds (because there is no opposition). Note that $R(t)$ is set to her own strategy $\left[R(t) \leftarrow a_{1}^{1}\right]$, securing average $b$ earnings per round from which $C_{R}$ must be subtracted. The optimal strategy is to intervene if $C_{R}<b / 2$.

In Table I of the main text, we show the case with two agents $[N(t)=2]$ and one game $(n=1)$. There are three scenarios worth considering separately: (i) No player intervenes (upper-left block matrix in Table I): $R(t)$ is set randomly; both players win half of the time. If they play the same strategy, whenever they win, they must split the earnings. Playing different strategies, each agent still wins half of the time but always keeps all the earnings. Thus, the model reduces to a minority game: It is preferable to play distinct strategies. (ii) Only one of the players attempts to intervene (off-diagonal block matrices in Table I): That agent pays $C_{R}$ and sets $R(t)$ to her own strategy. If only one agent is given the option to intervene, it always becomes favorable if $C_{R}<b / 4$, disregarding the other agent's behavior. If $b / 4<C_{R}<b / 2$, intervention is favorable only if both agents play different strategies. If $C_{R}>b / 2$, it never becomes favorable to intervene. (iii) Both agents attempt to rig the game (bottom-right block matrix in Table I): Both agents pay $C_{R}$, but they only succeed in setting $R(t)$ if they play the same strategy. This case turns the original minority game into a neutral one: If both attempt to rig the game, they always earn the same, regardless of whether their strategies match. This payoff is always less than the best scenario with no intervention-but, if $C_{R}<b / 4$, it is greater than the case with no intervention and matching strategies.

The payoff matrix for $N(t)=3$ and one game is split between Tables III-V. Note that either all players choose the same strategy or one differs. In such a case, we assume that agent 1 is in the minority (and we call her the minority player) without loss of generality. Agents 2 and 3 are called majority players.

Table III shows payoffs when only the minority player is allowed to intervene. If she does not intervene (left half of Table III), we are, again, dealing with a minority game. The Nash equilibria of this subgame $\left(r_{1}^{i}=0 \quad \forall i\right.$ and $a_{1}^{1} \neq a_{1}^{2,3}$, gray in Table III) are Nash equilibria of the whole game if $C_{R}>b / 4$. For cheaper rigging costs, the global Nash equilibria disappear as it becomes favorable for one of the majority agents to rig the game (Table IV). This prompts a dynamic situation similar to the one discussed in the main text. Finally, Table V shows both majority players successfully rigging the game. Interestingly, if all three agents try to manipulate $R(t)$ (red frame), the model turns into a majority game. With full intervention, an agent in the minority would earn nothing: Thus, full coordination is a Nash equilibrium of that subgame, which always happens if $N(t) \geq 3$; therefore, in general, when everybody is intervening, the minority game is turned into a majority one. However, full coordination is not a global Nash

TABLE II. Payoff matrix for one game with one player. The agent's behavior is coded by two bits. The first one ( $a$ ) indicates the agent's strategy ( 0 or 1$)$. The second bit $(r)$ indicates whether the agent attempts to rig the game or not.

\begin{tabular}{lcccc}
\hline \hline Player's behavior & $a=0, r=0$ & $a=1, r=0$ & $a=0, r=1$ & $a=1, r=1$ \\
\hline Payoff & $b / 2$ & $b / 2$ & $b-C_{R}$ & $b-C_{R}$ \\
\hline \hline
\end{tabular}

TABLE III. Payoff matrix of one game with three players-only the minority player can rig the game. We assume that player 1 is in the minority when there is no consensus. In this table, only player 1 is allowed to rig the game, so she always succeeds. Entries marked in gray are global Nash equilibria when rigging is very expensive $C_{R} \gg b$.

\begin{tabular}{|c|c|c|c|c|}
\hline $\begin{array}{ll}\text { Agent 2, } 3 & \text { Agent } 1 \\
\end{array}$ & $a^{1}=0, r^{1}=0$ & $a^{1}=1, r^{1}=0$ & $a^{1}=0, r^{1}=1$ & $a^{1}=1, r^{1}=1$ \\
\hline$a^{2,3}=0, r^{2,3}=0$ & $b / 6$ & $b / 4-b / 2$ & $b / 3 b / 3-C_{R}$ & ${ }_{0} b-C_{R}$ \\
\hline$a^{2,3}=1, r^{2,3}=0$ & $b / 4-b / 2$ & $b / 6$ & ${ }_{0} b-C_{R}$ & $b / 3 b / 3-C_{R}$ \\
\hline
\end{tabular}


TABLE IV. Payoff matrix of one game with three players - only one of the majority players (player 2) rigs the game. This is the only situation in which the symmetry between the majority players is broken.

\begin{tabular}{|c|c|c|c|c|}
\hline \begin{tabular}{|ll} 
Agent 2 & Agent 1 \\
\end{tabular} & $a^{1}=0, r^{1}=0$ & $a^{1}=1, r^{1}=0$ & $a^{1}=0, r^{1}=1$ & $a^{1}=1, r^{1}=1$ \\
\hline$a^{2,3}=0, r^{2}=1, r^{3}=0$ & $b / 3-C_{R} b / 3$ & $b / 2-C_{R} \quad 0$ & $b / 3-C_{R}$ & $b / 4-C_{R}^{b / 2-C_{R}}$ \\
\hline$a^{2,3}=1, r^{2}=1, r^{3}=0$ & $b / 2-C_{R} \quad 0$ & $b / 3-C_{R} b / 3$ & $b / 4-C_{R}^{b / 2-C_{R}}$ & $b / 3-C_{R}$ \\
\hline \begin{tabular}{|ll} 
Agent 3 & Agent 1 \\
\end{tabular} & $a^{1}=0, r^{1}=0$ & $a^{1}=1, r^{1}=0$ & $a^{1}=0, r^{1}=1$ & $a^{1}=1, r^{1}=1$ \\
\hline$a^{2,3}=0, r^{2}=1, r^{3}=0$ & $b / 3$ & $\begin{array}{ll}b / 2 & 0 \\
\end{array}$ & $b / 3 \quad b / 3-C_{R}$ & $b / 4 \quad b / 2-C_{R}$ \\
\hline$a^{2,3}=1, r^{2}=1, r^{3}=0$ & $\begin{array}{ll}b / 2 & 0 \\
\end{array}$ & $b / 3$ & $b / 4 \quad b / 2-C_{R}$ & $b / 3 \quad b / 3-C_{R}$ \\
\hline
\end{tabular}

TABLE V. Payoff matrix of one game with three players - both majority players rig the game. Since they are in the majority, they always succeed in their attempt to set the winning rule. If all three players rig the game simultaneously (red frame), the model turns into a majority game-i.e., the best strategy is to play what everybody else is playing. Gray squares indicate Nash equilibria of this subgame.

\begin{tabular}{|c|c|c|c|c|}
\hline $\begin{array}{ll}\text { Agent } 2 & \text { Agent } 1 \\
\end{array}$ & $a^{1}=0, r^{1}=0$ & $a^{1}=1, r^{1}=0$ & $a^{1}=0, r^{1}=1$ & $a^{1}=1, r^{1}=1$ \\
\hline$a^{2,3}=0, r^{2,3}=1$ & $b / 3-C_{R} \quad b / 3$ & $b / 2-C_{R} \quad 0$ & $b / 3-C_{R}$ & $b / 2-C_{R} \quad 0$ \\
\hline$a^{2,3}=1, r^{2,3}=1$ & $b / 2-C_{R}=0$ & $b / 3-C_{R} \quad b / 3$ & $b / 2-C_{R} \quad 0$ & $b / 3-C_{R}$ \\
\hline
\end{tabular}

equilibrium. If such coordination would happen, it pays off to any of the agents to stop trying to rig the game. Her earnings would still be guaranteed by the efforts of the others. This result suggests that our model reaches large levels of coordination (as discussed in the main text) in a tragedy-of-the-commons scenario.

In the cases just described, population is fixed and behaviors do not change. Hence, games are independent of each other, and we can use these payoff matrices to approximate averages over many games. The situation becomes more difficult as dynamics are included: Behaviors change as agents are renewed, and averages over time involve complex, recurrent feedback. For example, agent 1 may rig games to favor agent 3 who, in turn, is securing another game for agent 1 . Thus, the $R_{k}(t)$ become coupled with each other, resulting in much more complicated payoff matrices.

\section{APPENDIX B: SUPPORTING PLOTS AND DISCUSSION FOR INCREASING ECONOMY SIZE AND FIXED COMPLEXITY}

Despite its simplicity, the model turns out to have very rich dynamics. Its behavior changes, sometimes drastically, with the economy complexity (as measured by the number of games, $n$ ) or with its size (as measured by distributed wealth per round, $B$ ). We now expand the discussion for fixed complexity and growing economy size.

The simplest case with one game $(n=1)$ is summarized in Fig. 6, which shows the average population size in the steady state, rigging pressure, and strategy entropy as the economy size grows. Circles over these plots show values of $B$ for which we display 1000 iterations of the dynamics in Fig. 14 in Supplemental Material [26].

If $B$ is small, little wealth is distributed per round, and it is not favored to expend the few available resources in intervening with the economy. Thus, $R(t)$ is randomly set to 0 or 1 each time step, and the steady population does not settle for either strategy. The second row of Fig. 14(a) in Supplemental Material [26] shows $f_{1}$ (the fraction of agents with strategy $\left.a_{1}^{i}=1\right)$ over time, which fluctuates close to $f_{1}=0.5$. This results in a high strategy entropy $\left\langle h_{1}^{a}\right\rangle$, as shown in the third row of Fig. 14(a) in Supplemental Material [26]. The fourth row shows that, indeed, the rigging pressure is negligible for low values of $B$.

Above some amount of available resources, some rigging pressure builds up periodically [lower row of Figs. 14(b) and 14(c) in Supplemental Material [26]]. Let us assume a situation in which no agent is intervening. Then, a mutation results in a single agent that alters the game. She secures the next rounds played, and all agents playing her same strategy consequently benefit. The latter will have a slightly higher payoff since they do not expend $C_{R}$ in rigging the game. Darwinian dynamics amplify these slight differences exponentially, so the rigging agents will eventually be driven off to extinction. Some agents playing the minority strategy might have survived this process (perhaps because they had some savings), or they might reappear through mutation. After all rigging agents are 

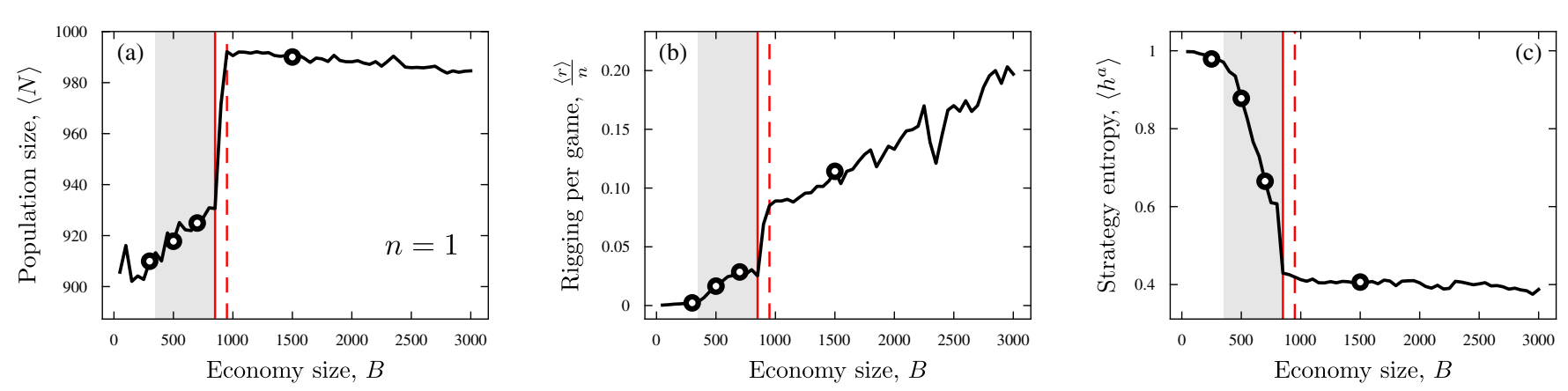

FIG. 6. Fixed economy complexity $n=1$ and growing distributed wealth $B$. (a) Average population size in the steady state. (b) Rigging pressure over the only game in the steady state. (c) Strategy entropy in the only game in the steady state. Circles over the curves indicate values of $B$ for which we show samples of the dynamics in Fig. 9 in Supplemental Material [26].
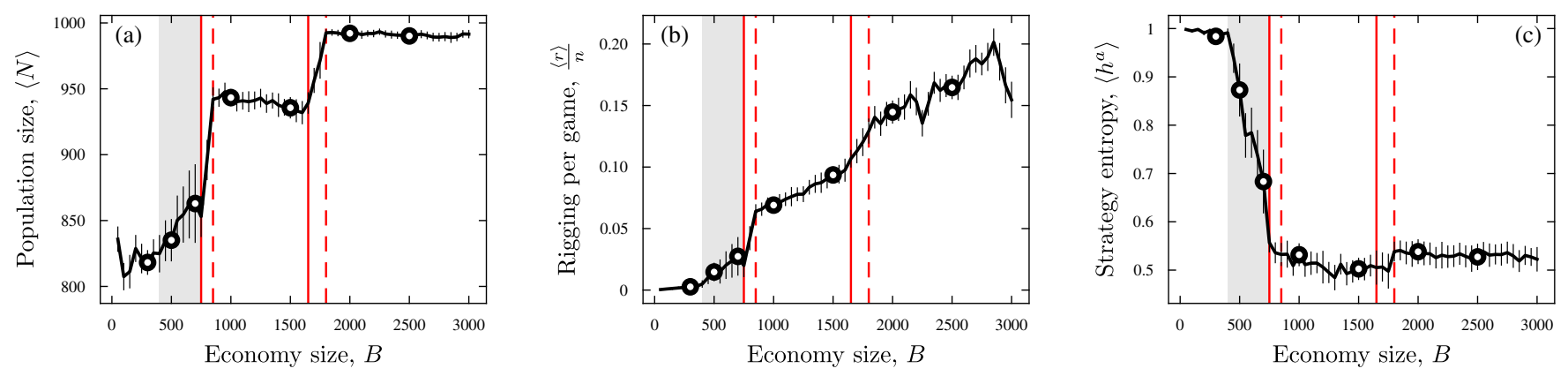

FIG. 7. Fixed economy complexity $n=2$ and growing distributed wealth $B$. (a) Average population size in the steady state. (b) Average rigging pressure per game. (c) Average strategy entropy over the two games. Circles over the curves indicate values of $B$ for which we show samples of the dynamics in Fig. 10 in Supplemental Material [26]. Error bars indicate the standard deviation over the last 500 iterations of the corresponding simulation.

removed from the population, those playing the minority strategy will have a greater payoff (since they share less of their earnings) and start making a come back. A mutation in their descendants might produce an agent rigging the game to further favor the minority, which might become the majority option over time, thus starting a cycle all over again. Figures 14(b) and 14(c) in Supplemental Material [26] show that this oscillating behavior takes place for a range of economy sizes $B$.

Vertical dashed blue lines in this figure show the point at which population reaches a maximum, which happens as the rigging pressure peaks as well. Interestingly, this is also the point at which the proportion of agents playing either strategy is well balanced. The population minimum (indicated by vertical dashed red lines) happens when the rigging pressure is minimal as well, and the population presents a more homogeneous strategy.

Increasing the economy size results in longer alternating cycles of this nature. Eventually, these cycles become infinitely long so that most of the population converges to a consensus strategy [Fig. 14(d) in Supplemental Material [26] ]. This homogeneous state supports a larger
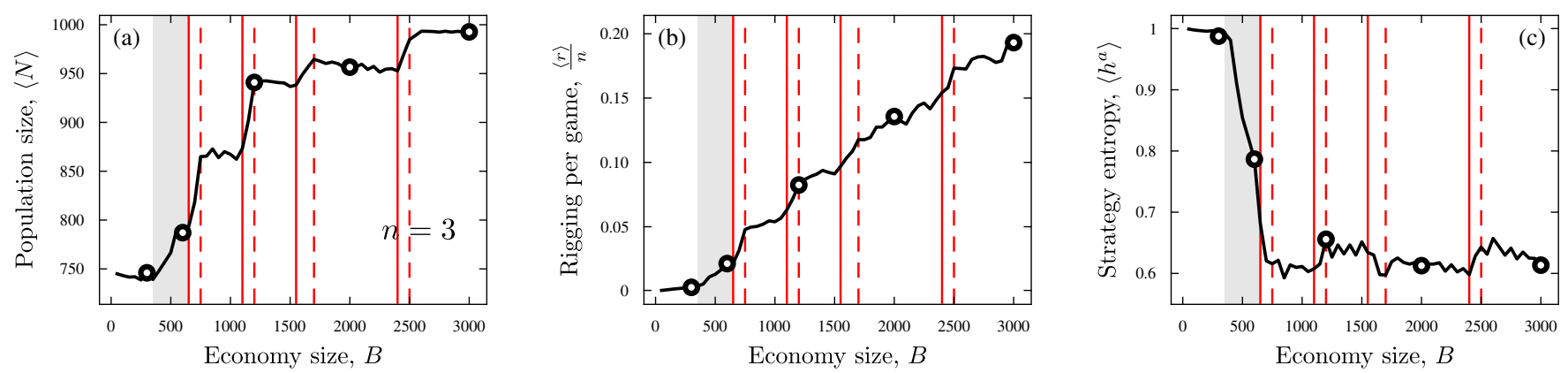

FIG. 8. Fixed economy complexity $n=3$ and growing distributed wealth $B$. (a) Average population size in the steady state. (b) Average rigging pressure per game. (c) Average strategy entropy over the three games. Circles over the curves indicate values of $B$ for which we show samples of the dynamics in Fig. 11 in Supplemental Material [26]. 

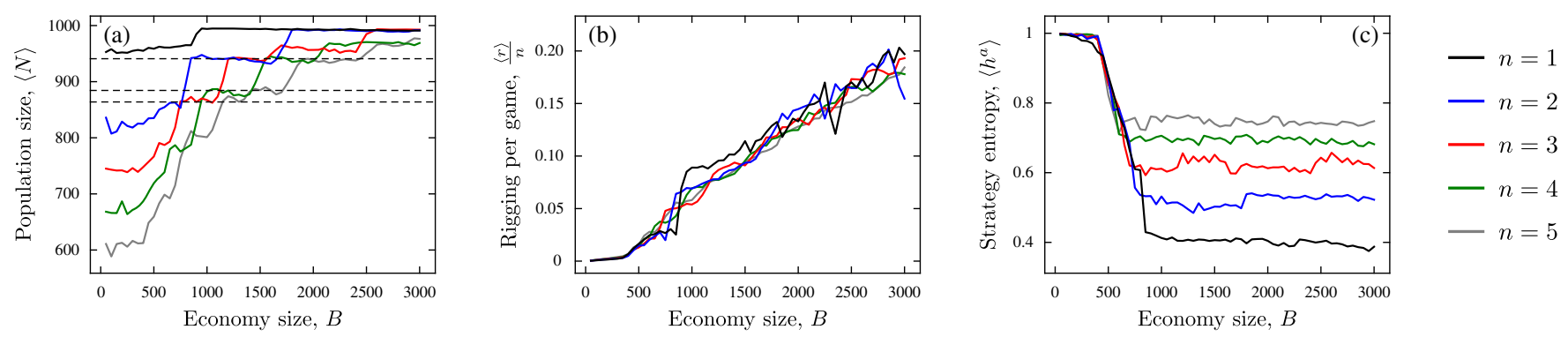

FIG. 9. Fixed economy complexity $n$ and growing distributed wealth $B$-comparison of cases with small complexity. (a) Average population size in the steady state. Horizontal dashed lines help us identify cases that, despite having different $n$ and $B$, reach similar stable population sizes, suggesting that some scenarios might be essentially equivalent. (b) Average rigging pressure per game. (c) Average strategy entropy over the $n$ games in each case.

population than the cyclic or fully random regimes. Convergence to the consensus is not full. A reservoir of minority agents survives, suggesting that they occasionally succeed in rigging the game and upsetting the majority.

We now extend the discussion of the case with $n=2$ games from the main text. Figure 7 reproduces the first three panels of Fig. 2 but with added error bars (1 standard deviation over the last 500 time steps) to illustrate typical fluctuations. Such fluctuations are comparable in other, similar plots (Fig. 6 for $n=1$ and Fig. 8 for $n=3$, as well as Figs. 9 and 10, which compare several $n$ ), where they are omitted for clarity. Figures 2 and 7 both show a transition (similar to the one observed for $n=1$ ) from unintervened games [sample dynamics are shown in Fig. 15(a) in Supplemental Material [26] ], through cycles of growing and declining coordination [Figs. 15(b) and 15(c) in Supplemental Material [26] ], to more homogeneous states [Figs. 15(d)-15(g) in Supplemental Material [26] ].

In the main text, we indicate that the cyclic regime is transited simultaneously for both games, but there are some nuances. Figures 15(b) and 15(c) in Supplemental Material [26] show that rigging pressure is synchronized for both games (bottom row), but this synchronization is broken when we look at the consensus in each game. For $B=500$ [Fig. 15(b) in Supplemental Material [26] ], on average, the population does not converge on persistent homogeneous strategies for either of the games. But the strategies do not stay divided roughly in half either [as it happens for
$B=300$, Fig. 15(a) in Supplemental Material [26] ]. For a larger economy size with cyclic behavior $[B=700$, Fig. 15(c) in Supplemental Material [26] ], on average, the population reaches a stable, broad consensus in one of the games, while agreement in the other game rises and falls. Both games take turns being the one with a consensus, but a simultaneous consensus in both games never lasts.

Further increasing $B$, the cyclic behavior is subdued, and two more regimes are revealed, each separated by a large boost in stable population size [Fig. 7(a)]. As noted in the main text, these last regime shifts are not accompanied by large changes in strategy entropy, $\left\langle h_{k}^{a}\right\rangle$. We conclude that within-game coordination has been exhausted and that new kinds of correlations, now across games, take place. Figures 15(d)-15(g) in Supplemental Material [26] sample the dynamics in those extra regimes. Panels (d) and (e) of Fig. 15 in Supplemental Material [26] sample between $B \simeq$ 800 and 1600, and panels (f) and (g) sample for $B>1700$. The second row shows large consensuses around the strategies of both games (a reservoir of minority agents always survives). The level of consensus (as indicated by $f_{k}$ and $\left\langle h_{k}^{a}\right\rangle$ ) remains similar in both regimes. We note that population size fluctuates more in the regime between $B \simeq$ 800 and 1600 , while the regime for $B>1700$ supports a larger, more stable population. Rigging pressure is also notably higher in this last case, suggesting a more efficient coordination between rigging efforts across games.
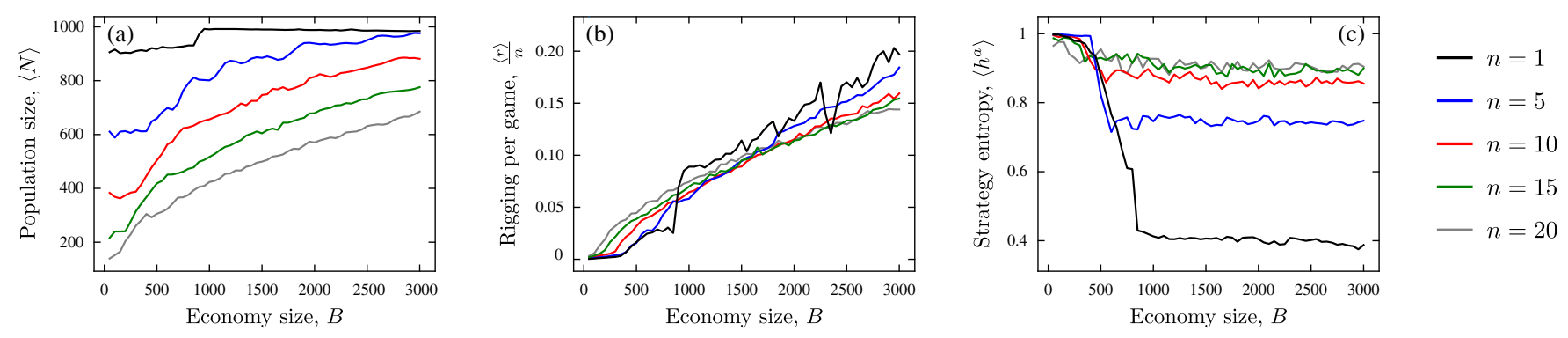

FIG. 10. Fixed economy complexity $n$ and growing distributed wealth $B$-comparison of cases with large complexity. (a) Average population size in the steady state. Boosts in stable population size are smoothed into a continuous buildup as complexity increases. (b) Average rigging pressure per game. (c) Average strategy entropy over the $n$ games in each case. 

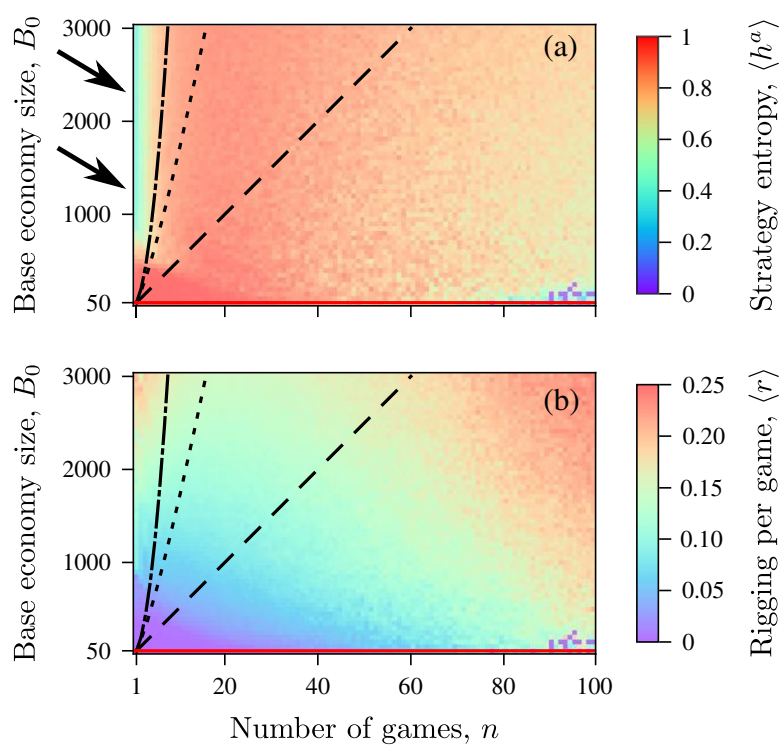

FIG. 11. Comprehensive maps of strategy entropy and rigging pressure. (a) Average strategy entropy $\left\langle h^{a}(t)\right\rangle$, showing a dent for small $n$ and large $B-$ a situation that we compared to cartels in the main text. This coordination regime falls apart swiftly as complexity grows a little bit. If we move to very large $n$ without increasing $B$, coordination starts to build up again-yet very smoothly. (b) Rigging pressure per game, $\langle r(t) / n\rangle$. Unsurprisingly, it grows with the amount of wealth distributed. More interestingly, it also grows with the number of degrees of freedom in the system.

This feature would allow the population to extract more wealth on average. For example, consider the reservoir of minority agents for each game. Those minority agents might not be the same for both games. A transition to a higher across-game coordination might happen if the agents playing the minority in both games become the same.

Finally, Fig. 8 summarizes different regimes for $n=3$ games. We observe more shifts (as identified by boosts in population size) than for $n=2$, which is compatible with more available across-game coordination options. Figure 16 in Supplemental Material [26] samples the dynamics for these various regimes. In the cyclic scenario [Fig. 16(b) in Supplemental Material [26] ], rigging pressure across all games again appears synchronized, while this synchronization is lost in the consensus around each game's strategy. In this example, two out of three games reach consensus simultaneously, and they take turns being in and out of consensus. For other values of $B$ (not shown), consensus was reached for at most one game. For larger $B$, the cyclic behavior is subdued again. Extra regime shifts (as revealed by boosts in $\langle N\rangle$ ) happen after within-game coordination has been exhausted, which is again consistent with the idea that more games bring in more possible across-game coordination.

Adding more games appears to have two different effects: On the one hand, more regimes seem to become available; on the other hand, more consecutive regimes seem to be visited within a smaller range of $B$. Thus, as we increase $B$, regimes progress more rapidly into each other (Fig. 9). This effect is exaggerated if even more games are available (Fig. 10) — so much so that, instead of regime shifts, we approximate a continuous progression. The increase in rigging pressure per game becomes parsimonious as well (while for a small number of games it presents a few discrete boosts associated with regime shifts).

Figure 9(c) shows that exhausting the within-game coordination results in a drop of strategy entropy, $\left\langle h^{a}\right\rangle$. We see that this drop becomes less accentuated for larger $n$ [Fig. 10(c)], which suggests that the onset of interactions across games thwarts within-game coordination. In other words, a more complex economy seems to enable populations with more diverse strategies within single games.

\section{APPENDIX C: ROBUSTNESS OF RESULTS AGAINST MODEL VARIATIONS}

Similarly to the maps built for $\langle N\rangle$ and $\sigma(N) /\langle N\rangle$ in Fig. 4, we can chart other quantities such as average strategy entropy $\left[\left\langle h^{a}\right\rangle\right.$, Fig. 11(a)] and the rigging pressure per game $[\langle r\rangle / n$, Fig. 11(b)]. These maps reveal the model's regimes, but how general is the uncovered phenomenology? Does it depend critically on model parameters?

We have six independent parameters: The first one sets $n$, the number of games-thus the economy's complexity. The second one sets the economy size (all $b, B$, and $B_{0}$ become fixed by setting one of them and the number of games). Two additional parameters set up costs (of attempting to rig a game, $C_{R}$; and of producing descent, $C_{C}$ ). Finally, we have the mutation rate $p_{\mu}$ and $N^{\max }$, which sets an external upper limit to the population. We designed our model with the hope of pinning down essential features of rigged economies. We hope that the elements involved in the model introduce as few additional effects as possible. In that sense, an abundance of parameters is not desired. Furthermore, we hope that the most interesting phenomenology depends on $B$ and $n$, which are at the center of our research questions. Toying around with variations on model parameters, we have found that the observed phenomenology is robustly reproduced, which is strongly in favor of the minimalism of our approach. Let us look at some model variations in action:

First, we note that the cost of rigging a game $C_{R}$ sets a scale with respect to the wealth allotted to each game $b=B / n$. In our simulations, we set $C_{R}=1$. If we try a different value of $C_{R}$, we could normalize $\tilde{b} \equiv b / C_{R}$ and $\tilde{C}_{C} \equiv C_{C} / C_{R}$, thus remapping our model back to a case with $\tilde{C}_{R} \equiv C_{R} / C_{R}=1$. Hence, we just need to explore three free parameters others than $n$ and $B$.

Figure 12 shows what happens to $\langle N\rangle$ and $\sigma(N) /\langle N\rangle$ as $C_{C}$ changes. The effect in $\langle N\rangle$ seems negligible for the values explored [Figs. 7(a)-7(c)]. More notably, this 

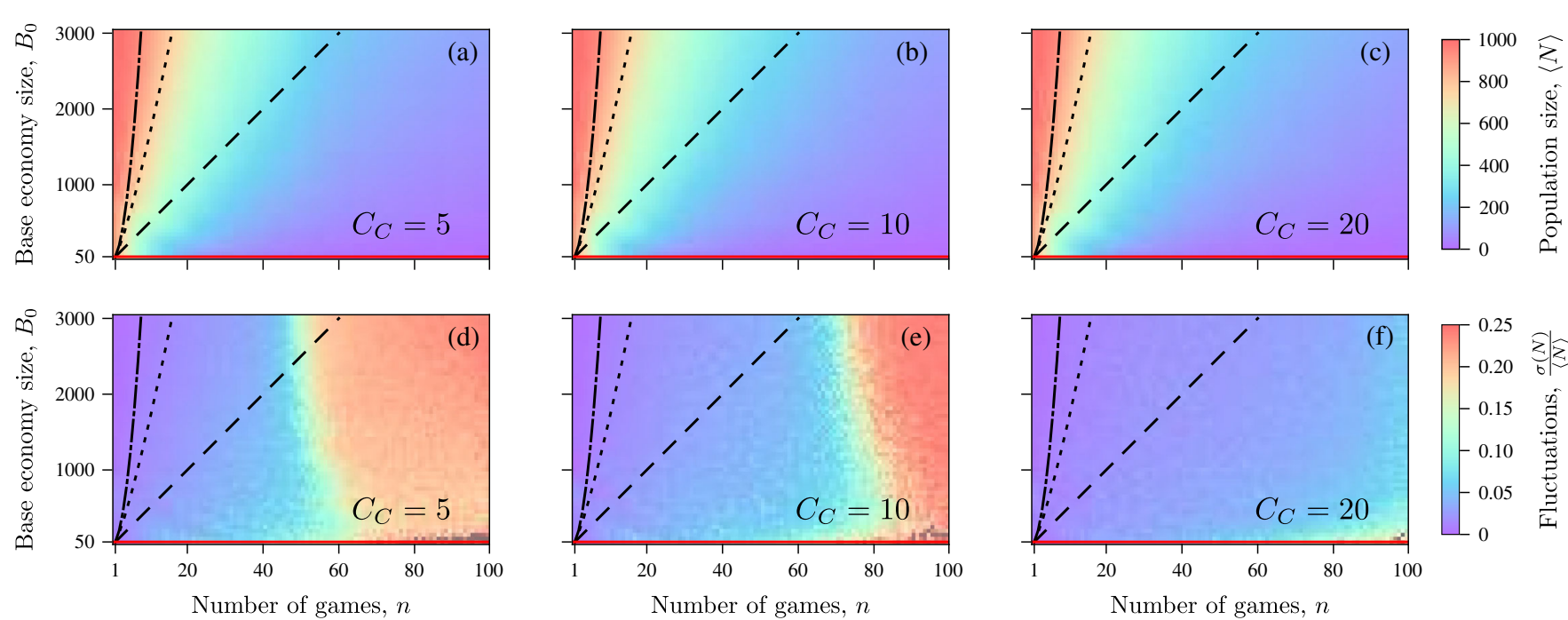

FIG. 12. Comprehensive maps of $\langle N\rangle$ and $\sigma(N) /\langle N\rangle$ for varying replication cost $C_{C}$. Maps result from simulating case I (i.e., $\left.B_{I}=B_{0}, b_{I}=B_{0} / n\right)$ for ranges of economic complexity $n$ and distributed wealth $B_{0}$. Black curves represent trajectories $B=B\left(B_{0}=\right.$ $50, n)$ for cases II, III, and IV (dashed, dotted, and dot-dashed, respectively). Horizontal red lines at the bottom of each map represent case I with $B_{0}=50$. Panels (a)-(c) show $\langle N\rangle$, and panels (d)-(f) show $\sigma(N) /\langle N\rangle$. (a,d) $C_{C}=5$; (b,e) $C_{C}=10 ;(\mathrm{c}, \mathrm{f}) C_{C}=20$.

parameter displaces the onset of the large-fluctuations regime [Figs. 7(d)-7(f)]. If $C_{C}$ is smaller, this regime ensues for a lower economy complexity $n . C_{C}$ tells us how cheap it is to have descendants. When it is cheaper, it is easier to trigger large fluctuations-a large descent explores more behaviors simultaneously, as well as displacing a bigger proportion of former agents. Both of these actions result in major disruptions of $R(t)$. Thus, cheaper descent more easily brings up a scenario in which agents are continuously deceiving each other into bankruptcy. If this is correct, other parameters that promote behavior diversity or population renewal should displace the onset of the large-fluctuations regime in a similar manner.

We ran simulations (not shown) to check how varying the mutation rate $p_{\mu}$ prompts this expected outcome, too. Indeed, increasing $p_{\mu}$ advances the large-fluctuations regime, as expected from a parameter that increases behavioral diversity. Other variables (rigging pressure and strategy entropy) behave as reported above as either $C_{C}$ or $p_{\mu}$ are changed. Importantly, the consensus for low $n$ and large $B$ is reproduced; and this consensus is also prevented as complexity is increased.

The model parameter $N^{\max }$ sets a maximum population size, which makes sense as an external constraint—e.g., the amount of people that can occupy a territory. It could also be seen as a manufactured (rigged) limit to participants in a market. This kind of economic manipulation is important, but we do not address it here; thus, it should be studied in the future. Note that $N^{\max }$ also parsimoniously induces a finite lifespan. If $N^{\max } \rightarrow \infty$, agents who do not attempt any rigging never pay $C_{R}$ and never end up with negative wealth and hence never die. This situation is unrealistic and undesired. Through $N^{\max }$, long-lasting agents are naturally and randomly replaced by newborns. We explored model alternatives without $N^{\max }$ (e.g., enforcing a maximum lifetime), and the same phenomenology has been robustly recovered.

We ran additional simulations with $N^{\max }=100,5000$, and 10000 (the latter took more than ten days in a fairly powerful cluster). These tests resulted in qualitatively similar overall structures of the $\langle N\rangle$ and $\sigma(N) /\langle N\rangle$ charts (not shown). Quantitatively, the onset of the largefluctuations regime changed as expected. Increasing $N^{\max }$, this regime starts for notably larger values of $n$. A lower $N^{\max }$ results in an earlier onset of large fluctuations. Above, we argued that the onset of this regime was advanced by (i) mechanisms that result in more diverse strategies competing closer together or (ii) a higher population turnover. Thus, lower $C_{C}$ (cheaper reproduction) and higher $p_{\mu}$ (increased mutation) both advance the onset of the regime. A larger $N^{\max }$ has the effect of diluting the competence. Oppositely, smaller $N^{\text {max }}$ results in tighter competence.

Regarding the other relevant regime, plots of strategy entropy for different $N^{\max }$ (not shown) again indicate how consensus is reached when enough wealth is present and the number of games is small. This consensus, again, breaks apart swiftly as the number of games is increased.

Some modeling choices might introduce ad hoc results independent of model parameters. We can never be exhaustive, but we have tried some model alternatives to make sure that our results are robust. Some of the variants extend the model in ways that might allow us to tackle new questions around rigged economies in the future. The variations we explored include the following: (i) We eliminated $N^{\max }$. Instead, we set a fixed chance that 
newborn agents would substitute an older one (otherwise, they were appended to the population pool, making it grow). In such model variation, population might grow unboundedly or reach a steady state. In another alternative to suppress $N^{\text {max }}$, we never replaced older agents at all. We prevented unbounded growth by setting a maximum lifespan (agents who reached it were removed). (ii) We explored biased games and games with momentum. In the former, one strategy was favored. More agents were needed to rig the game against it. In the latter, the bias would shift to favor the last winning strategy (i.e., once an option is preferred, it becomes more difficult to overturn). (iii) We broke the symmetry between games by (i) making some of them more profitable than others (see below) or (ii) cheaper to rig than others, and (iii) connecting games in a dependency network, thus affecting each other's winning strategies. (iv) We allowed agents to choose what games to play. These model variations will be reported in future papers-as some of them illuminate questions beyond the scope of this work. In all of these variants, we reliably observed the main regimes discussed in this paper (i.e., large fluctuations for large $n$, and consensus for wealthy, simple economies that breaks apart swiftly as complexity increases). The exact onset and size of large fluctuations (as a function of $n$ ) and some quantitative aspects of the consensus regime depended on the model variation and associated parameters. But, qualitatively, our results are very robust, thus strengthening the idea that the uncovered phenomenology should show up for other models of rigged economies.

One of these variants provides a nuance to one of our results. Let us have a closer look at it:

Our original model assumes an extreme symmetry: All economic games are equivalent-all of them are equally costly to rig and report the same benefit if the correct strategy is played. But real-world economies present a variety of assets into which to invest, jobs to perform, deals to strike, etc. Statistical distributions in economics are usually heavy tailed, meaning that outliers often dominate the scene. In terms of games, this means that some of them are overwhelmingly much more important than otherse.g., because they deal out much more wealth.

Given a fixed economy size $B_{0}$, let the $k$ th game deal out an amount of wealth $b_{k}$ with the constraint $\sum_{k} b_{k}=B_{0}$. We tried random, uniformly distributed $b_{k}$, obtaining results similar to the original model. This result is expected: While some fluctuations are introduced, a uniform distribution does not present great biases towards any game - they all turn out to be, on average, fairly equivalent. To break this symmetry, we explore starkly biased distributions for $b_{k}$. Let one of the games deal out $90 \%$ of all wealth. Without loss of generality, let this be the first game-thus, $b_{1}=0.9 B_{0}$ and $b_{k}=$ $0.1 B_{0} /(n-1)$ for all $k \neq 1$.

Figure 13(a) shows that the large-fluctuations regime still exists for this scenario. For case II (economy size grows linearly with economic complexity), the size of fluctuations is quite similar to the original model, and their onset occurs
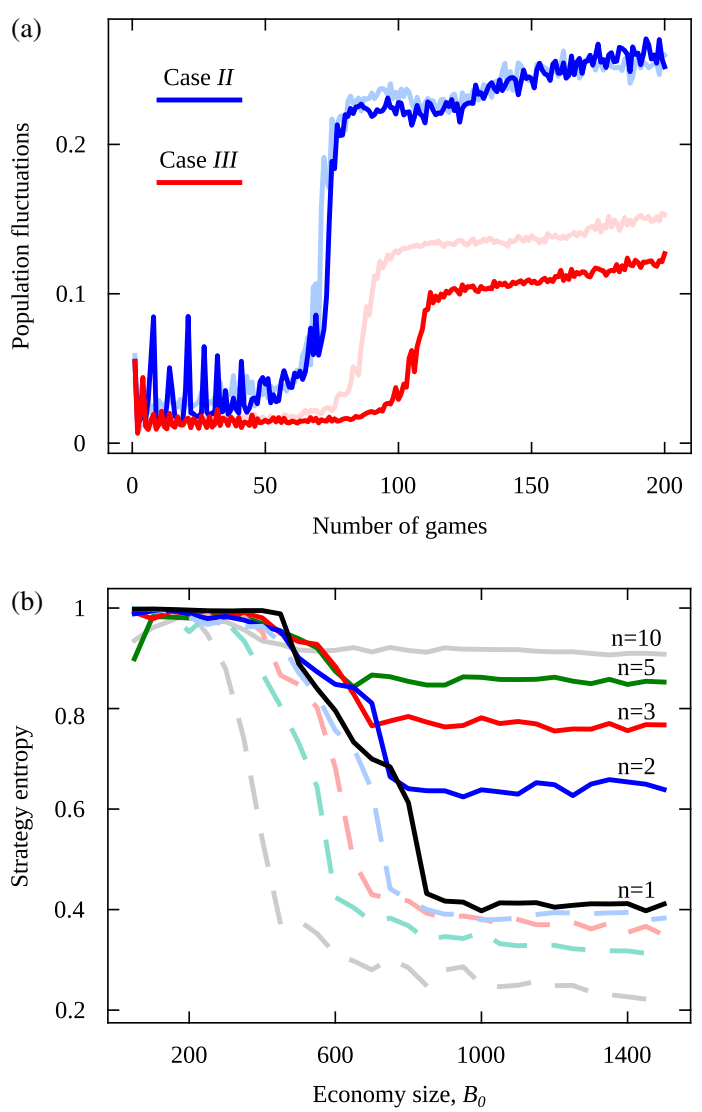

FIG. 13. Charting a model of rigged economic games of different importance. In these simulations, one game deals out $90 \%$ of the wealth in each round. All other games deal out the rest of the wealth in equal amounts. (a) Illustration of the largefluctuations regime for cases II and III. For comparison, lighter colors show the same curves from the original model. (b) Illustration of the regime with relatively broad consensus. Dark colors indicate the average of strategy entropy across all games (including the main one). Light colors indicate strategy entropy for the main game.

for a complexity just slightly higher. For case III (economy size grows faster than linearly with economic complexity), compared to the original model, large fluctuations are smaller, and their onset happens for fairly higher complexity than for the original model. We speculate that the stark asymmetry results in an effective number of games $\tilde{n}<n$, suggesting an explanation as to why the onset of large fluctuations happens at higher complexity. The gross amount of wealth in the system seems to be a relevant factor as wellas the differences between cases II and III show. Note that the first game handles $90 \%$ of the wealth; it is interesting that the $(n-1)$ remaining games can throw the system in the largefluctuations regime while controlling just $10 \%$ of the wealth.

Figure 13(b) shows the broad consensus regime for wealthy, yet simple economies. We find a counterintuitive result with implications for the strategy of increasing complexity to defuse cartels. Solid, dark-colored curves plot strategy entropy averaged over all games (including the 
first one). This entropy becomes larger with $n$, and it does so faster than in the original model [compare to Fig. 9(c)], suggesting that the consensus is more easily abandoned. However, looking at the strategy entropy of the first game alone (dashed, light-colored curves), the consensus around this game actually grows as the economy becomes more complex. Thus, in order to defuse consensus, the degrees of freedom added to our economy must be of relevance. Adding rather low-paying games might have the opposite effect-reinforcing the main consensus.

There are other possibilities to model asymmetrically rigged games. For example, we set up the $b_{k}$ to follow Zipf's law such that the $k$ th game would deal out $b_{1} / k$ wealth per round (always with $\sum_{k} b_{k}=B_{0}$ ). In this version, we observed less differences (with respect to the original model) regarding large fluctuations, but we again found the strengthened consensus around the first game illustrated in Fig. 13(b). Alternatively, some games could be cheaper to manipulate than others. Actually, if all games are equally costly to rig (despite some of them having much lower $b_{k}$ ), it might contribute to the robustness of large fluctuations. Finally, we can conceive that games depend on each other, such that the winning strategy in one of them affects the best strategy in others. A network of influences between games is another way to introduce asymmetries. Results from these variants will be presented in future studies.

\section{APPENDIX D: FURTHER DISCUSSION OF REAL-WORLD RIGGED ECONOMIES}

In Sec. III E, we discussed the GME short squeeze from the viewpoint of rigged games. It was intended as an exercise to find differences and similarities with a real-world case with apparent market manipulation. The main goal of our model is to uncover large-scale trends that might emerge as economy size and complexity grow. The phenomena uncovered should be seen as limit cases and a starting point from which to proceed towards more realism. Aiming at modeling actual markets, the symmetry between games will likely be broken (beyond what we just explored), as will the symmetry between agents (introducing different allowed behaviors, restricting the access of some agents to certain games or strategies, etc.). To achieve this goal, the approach in Ref. [19] (in which payoff matrices are less restricted), combined with ours, should be much more useful. In this Appendix, we explore a series of additional situations in which the economy appears to be rigged. In each case, we try to locate relevant variables or possibilities, some enabled by new technology. Alongside, we try to figure out how to tackle these issues with our approach.

\section{Massively accessible markets through trading apps}

We mentioned the unusual communication channels through which the GME short squeeze was organized
(Reddit or Twitter). Furthermore, GME went viral, promptly reaching huge audiences. Another important ingredient was the widespread use of trading apps. These platforms are truly disruptive, making very complex financial products available to a large body of (often unexperienced) investors. Thus, the engagement with such products becomes incredibly simple and cheap.

Traditional brokers trade mostly with complete shares, which might be prohibitive for small investors if their price is too high. Trading apps offer fractional shares and contracts for differences (contracts within the platform to bet that a commodity will gain or lose value but through which the investor never actually acquires the asset), which enables very low entry points. From a rigged games perspective, in a situation such as the GME short squeeze (in which buying stock also "manipulates" the winning strategy), the simplicity and inexpensiveness of trading apps lowers the rigging $\operatorname{cost} C_{R}$ in our model. These platforms usually offer leverage mechanisms as well. They lend money to investors so they can make a greater return on their positions-with a much higher exposure, too. We can model this as nonlinear effects of trading options. Agents would choose how much to invest in their "rigging" (i.e., we move beyond one agent, one vote), and this investment could be weighted nonlinearly.

On top of these financial aspects, trading apps often look like social networks. Some of them showcase their users' portfolios, making it simple to follow, copy, or merge successful profiles. These options come as close as possible to implementing our evolutionary dynamics or to how successful rules are copied in the El Farol problem. Social network dynamics are thus embedded directly into stock markets-potentially at a massive scale. Viral memes, polarization, or echo chambers might become relevant. Popular profiles might act as a "coarse graining" of the assets in their portfolio, introducing correlations between unrelated products. Widespread correlation, as we know, can trigger market crashes and might be undesired [23].

This social network structure adds interesting possibilities from the rigged games viewpoint. "Influencer" accounts could be traded (popular Twitter or Instagram profiles are already marketed for targeted advertising). Successful small investors could be "bought" to tilt stocks in a given direction. However big an influencer might become, officially they are small investors without much liability. Current regulations might become blurred, allowing these users some behaviors that are forbidden for professional brokers. As a case in point, the Reddit user Keith Patrick Gill (Ref. [50], one of the GME short-squeeze leaders) opened his testimony in front of the U.S. Congress with the following claim: "A few things I am not: I'm not a cat. I'm not an institutional investor, nor am I a hedge fund." He then proceeded to argue that his posting in social media, however influential, did not constitute investment advice. His testimony triggered yet another surge of GME 
trade. This case can be incorporated in our model through networks of influences between agents, which would affect the strategies and rigging choices of each other.

\section{Cryptocurrencies and internal complexity of economic games}

Cryptocurrencies are a favorite asset in trading apps. They were also heavily involved in the GME short squeeze: Bitcoin and Dogecoin became a refuge for small investors when numerous platforms halted the trading of GME [1]. The issues around social dynamics just discussed became relevant as small investors became coordinated online-but also, notably, as Elon Musk endorsed Dogecoin through Twitter. Given his reach and influence, with that single act, he might have tilted a whole game in his preferred direction at a very low cost.

Cryptocurrencies were created to articulate decentralized financial relationships, which might make them more vulnerable to manipulations similar to the ones in our model (i.e., emergent and, like this technology, decentralized). The financial protocols defining certain cryptocurrencies (e.g., Dai [51]) are literally voted on by holders of "governance tokens," which comes as close as possible to our model. Similar decision systems exist in more traditional institutions (e.g., shareholders vote on a company's strategies), but decisions are now made online, which is faster and within a less centralized structure; they can alter the very design and behavior of the currency.

The technology behind cryptocurrencies has been expanded to create smart contracts, non-fungible tokens (NFT), and potentially any imaginable financial product. Besides some very loose standards, the behavior of these products is programmed by their designers. This program is literally computer code that defines what each product does. For example, NFTs are non-fungible because a piece of code dictates that there is only a unique instance of each token and that they are not interchangeable. Other behaviors of these products might include the following: automated transactions of cryptocurrencies and other assets, generation of obligations, calls to other smart contracts, etc. Since their code is relentlessly executed, smart contracts are enforced in a decentralized manner.

Cryptocurrencies and derived products offer economic games in which people might decide to engage. The consequence of their behavior being programmed in a code is that changing this code is equivalent to changing the payoff matrix of the game. Changing the code is usually, but not necessarily, prevented. Writing code for these kinds of products can be thought of as a sort of "economic game engineering," which also implies that bugs in the code translate into bugs in the payoff matrix. These are unexpected degrees of freedom that can also be exploited to harness the flow of wealth in a specific direction-just as we explore in this paper. This exploitation of a badlyegineered code is precisely what happened to the short-lived Decentralized Autonomous Organization (DAO) [52,53]. DAO was institutionalized as a selfregulated venture capital organization. The way in which DAO was supposed to work was hard-coded into its smart contract, which binded its participants. After raising about 150 million dollars in a matter of weeks, a bug in the code enabled about 50 million dollars worth of Ether (the cryptocurrency of the Ethereum platform, on which DAO ran) to be diverted to a hacker's wallet. This case is by no means unique [54].

Using simple models such as ours, we conjecture that we could extract valuable insights about expected bugs in complex payoff matrices. For instance, say we quantify the complexity of smart contracts (e.g., through their number of lines of code, functions, or recursive calls, etc.). Then, given contracts of a certain complexity, what is the likelihood that they contain a harmful bug? What is the likelihood that it will be exploited by any of $N$ user agents? What is the cost of fixing such bugs? Note that changes to some parts of the code might affect others, potentially triggering avalanches of changes across the payoff matrix (as beautifully captured by Per Bak's avalanche model for similar complex systems [40]).

While our model captures external complexity, this new problem would require us to model internal complexity. An economic game is not an atomic, irreducible object; instead, it contains distinct mechanisms that make it work, and each of them might be manipulated to rig the game's outcome. How stable are these systems as their number of internal, "riggable" degrees of freedom grows? And as the wealth dependent on these mechanisms changes? And as the number of users (and potential "riggers") grows? Should economic games based on cryptotechnology guarantee a collateral that grows with their complexity to ensure a stable value? Should this collateral grow linearly or faster with complexity? To address these questions, we could apply our approach, only now inwards, towards the internal complexity of each game. For example, in this paper, each game deals out earnings independently if each right strategy is played. Instead, to tackle internal complexity, agents might need to play all (or a majority of) the correct strategies to earn the profits of a complex game. How does this alter the evolutionary dynamics of our model?

Like other software projects, the crypto ecosystem reuses pieces of code continuously. Thus, the internal complexity of a game might be replicated or exapted, creating a literal network of dependencies. For example, both Dai and DAO run on Ether. Manipulation of the Ethereum network (which might be as simple as overloading it to slow down transactions) might affect several tokens. These aspects could be incorporated into our model as networks of games affecting each other.

\section{Value of information}

Many classic schemes of market manipulation during the 20th century can be tracked down to espionage, leakage of 
merger agreements, or plain insider trading. In all of these cases, we identify information as the key asset. The owners of privileged information could anticipate market movements, knowing that a certain stock was about to increase or drop, and thus play the correct strategy. Information pervades all other aspects of economy and has been amply discussed in the literature [55-57].

Can we modify our approach to better account for access, use, and abuse of information? As the model stands, there are three relevant information sources, which constitute degrees of freedom that can be harnessed and exploited. The most obvious and less certain one is the winning rule when no intervention is taking place. The other two sources are the strategies and rigging choices wielded by other agents in the system. Let us take a closer look at each of these.

When it is not manipulated, our winning rule is a uniform, random variable with a value of 0 or 1 . This variable represents an unpredictable external signal that dictates how wealth will flow. In realistic setups, this variable could represent movements in a stock market. Then, while its direction ( 0 or 1 ) would emerge out of human activity, it should remain unpredictable, provided we have ideal and fair market conditions. Access to privileged information (as in classic insider trading) would reveal this signal's value before the market could react to it. The informed agent could correlate her strategy with the likely movement of the market. Unlike in our model, this intervention leaves the winning rule unaltered (and unpredictable for other agents). We would not incur the $C_{R}$ cost, but information might be expensive to obtain; there is also a risk of being penalized by regulators.

From the game-theoretic perspective, it is easy to revise our payoff matrices to include these effects. Assuming that no other form of rigging takes place (i.e., winning rules are set at random), we should include an agent whose strategy often correlates with that winning rule, thus altering the likelihood that she and others need to share the earnings. From the agent-based model perspective, we could allow "insider" agents to change their strategy after reducing the uncertainty around the winning rule [e.g., let them know that $R_{k}(t)=1$, with a 0.8 chance]. In both cases, the costs and risks just mentioned (of gathering information and being penalized) should be factored out from the payoff. What might the interplay be between both sources of market manipulation (the one explored in our model and the one related to privileged information)? Altering the payoff matrix of a game might be unfeasible or very costly; thus, such an intervention might not be convenient if the agent can, instead, change her strategy to the correct one, nevertheless. Under what combination of costs and penalties is one form of manipulation preferred over the other?

The other two sources of information in the model are the strategies and rigging choices of each agent. As our model functions, this information can be exploited in evolutionary terms, as correct responses are slowly selected for. For example, assume several agents are rigging a game in the same direction. For any of them, it would be convenient to stop their intervention attempt as long as at least one of the others keeps rigging the game in the desired way. Over evolutionary time, new agents with such mutation are more likely to persist. But we do not want to wait an evolutionary time. We wish to react immediately-e.g., to position ourselves in the minority if we know that the market is unrigged. Wolpert and Grana's work on whether to change a payoff matrix [19] comes closer to modeling this situation. In one of their model variations, agents might change their strategy because they suspect that a game has been meddled with.

\section{Pareto-rigged games}

The financial field of mergers and acquisitions offers a last example of market manipulation that suggests further, useful extensions for our model. This field is ripe with tactics, deceptions, and an array of strategies wielded by any side to reach a favorable agreement (e.g., for a buyer, to acquire a company at a price below its market value). Any of these tactics might deserve a lengthy discussion in terms of rigged games. We focus on the ploy popularly known as "greenmailing."

Greenmailing starts as a corporate raid in which a buyer seeks to acquire a target company-often one that works inefficiently or whose assets might be stripped by the buyer for profit. The buyer might also threaten to replace employees and managers. Once the buyer's position is strong and the threat becomes evident, the owners and leaders of the target company might feel compelled to react, for which they need to acquire stock (now held by the buyer) at a price well above market value. Rather than rigging an existing game, the buyer "engineers" an economic scenario for its own profit. Why does this tactic work? Why would the company's leadership not allow the buyer to acquire it at the market's trade value, thus leaving the buyer stuck with an allegedly inefficient business? What are the degrees of freedom that the buyer exploits in this engineered game?

The threat in greenmailing is to strip the target company of its assets and replace its managers, who are often stakeholders as well. These assets and positions (jobs) are valued differently by the market, the buyer, and the company leadership (who might have an emotional attachment as well). For the buyer, managers can be cheaply replaced. But this would affect the replaced employees in ways that the market cannot fairly factor in. The conflict arises from measuring the operation with different utility functions that cannot be compared to each other-issues of Pareto optimality enter the scene.

In our model, we could include games that deal out different assets that cannot be compared to each other, which each agent values differently. An agent's performance would not be measured by an absolute wealth 
anymore. Instead, each agent would rely on its utility function to determine her strategy in a given game, her choice to attempt to rig that game, and her ability to have descent. Within our model's evolutionary setup, it is possible to incorporate tools from multi-objective genetic algorithms for Pareto optimality [58-60]. Assets that are not convertible into each other add a new dimension of complexity that is strictly different from the number of games that we explore in this work.

[1] Wikipedia, Game Stop short squeeze, https://en.wikipedia .org/wiki/GameStop_short_squeeze.

[2] J. Zweig, The Real Force Driving the GameStop Revolution, https://www.wsj.com/articles/the-real-force-drivingthe-gamestop-amc-blackberry-revolution-11611965586\# selection-3170.0-3170.3.

[3] J. Tirole, The Theory of Industrial Organization (MIT Press, Cambridge, MA, 1988).

[4] R. D. Willig and R. Schmalensee, Handbook of Industrial Organization (Elsevier, New York, 1989).

[5] J. J. Laffont and J. Tirole, A Theory of Incentives in Procurement and Regulation (MIT Press, Cambridge, MA, 1993).

[6] M. Lewis, Flash Boys: A Wall Street Revolt (W. W. Norton Company, New York, NY, USA, 2015).

[7] J. A. Schumpeter, Capitalism, Socialism and Democracy (Routledge, Oxfordshire, England, United Kingdom, 1942).

[8] N. Ferguson, The Ascent of Money: A Financial History of the World (Penguin, City of Westminster, London, England, 2008).

[9] G. J. Stigler, The Theory of Economic Regulation, Bell J. Econ. Manage. Sci. 2, 3 (1971).

[10] J. Tirole, Hierarchies and Bureaucracies: On the Role of Collusion in Organizations, J. L. Econ. Org. 2, 181 (1986), https://heinonline.org/HOL/LandingPage?handle= hein.journals/jleo2\&div=16\&id=\&page $=$.

[11] J. J. Laffont and J. Tirole, The Politics of Government Decision-Making: A Theory of Regulatory Capture, Q. J. Econ. 106, 1089 (1991).

[12] D. Acemoglu, S. Johnson, and J. A. Robinson, Institutions as a Fundamental Cause of Long-Run Growth, in Handbook of Economic Growth 1, edited by P. Aghion and S. Durlauf (Elsevier B.V., Amsterdam, Netherlands, 2005), pp. 385-472.

[13] D. Acemoglu, S. Naidu, P. Restrepo, and J. A. Robinson, Democracy Does Cause Growth, J. Polit. Econ. 127, 47 (2019).

[14] M. Draghi, Sovereignty in a Globalised World, Speech on the Award of Laurea Honoris Causa in Law, University of Bologna, 2019.

[15] P. Barucca, A Fair Governance: On Inequality, Power and Democracy, Topoi 2020, 1 (2020).

[16] P. Milgrom and C. Shannon, Monotone Comparative Statics, Econometrica 62, 157 (1994).

[17] M. R. Caputo, The Envelope Theorem and Comparative Statics of Nash Equilibria, Games Econ. Behav. 13, 201 (1996).
[18] D. Acemoglu and M. K. Jensen, Aggregate Comparative Statics, Games Econ. Behav. 81, 27 (2013).

[19] D. Wolpert and J. Grana, How Much Would You Pay to Change a Game Before Playing It?, Entropy 21, 686 (2019).

[20] H. Gintis, Game Theory Evolving: A Problem-Centered Introduction to Modeling Strategic Behavior (Princeton University, Princeton, NJ, 2000).

[21] D. Whitehead, The El Farol Bar Problem Revisited: Reinforcement Learning in a Potential Game, ESE Discussion Papers, 186 (2008), https://www.pure.ed.ac.uk/ws/ portalfiles/portal/20037196/The_El_Farol_Bar_Problem_ Revisited.pdf.

[22] M. Buchanan, The Social Atom: Why the Rich Get Richer, Cheaters Get Caught, and Your Neighbor Usually Looks Like You (Bloomsbury Publishing USA, New York, NY, USA, 2008).

[23] D. Sornette, Why Stock Markets Crash: Critical Events in Complex Financial Systems (Princeton University Press, Princeton, NJ, USA, 2017).

[24] A. Devitt-Lee, H. Wang, J. Li, and B. Boghosian, A Nonstandard Description of Wealth Concentration in LargeScale Economies, SIAM J. Appl. Math. 78, 996 (2018).

[25] J. Li, B. M. Boghosian, and C. Li, The Affine Wealth Model: An Agent-Based Model of Asset Exchange That Allows for Negative-Wealth Agents and Its Empirical Validation, Physica (Amsterdam) 516A, 423 (2019).

[26] See Supplemental Material at http://link.aps.org/ supplemental/10.1103/PhysRevX.11.031058 for Figs. 9111.

[27] B. E. Hilbig and I. Thielmann, Does Everyone Have a Price? On the Role of Payoff Magnitude for Ethical Decision Making, Cognition 163, 15 (2017).

[28] A. Kajackaite and U. Gneezy, Incentives and Cheating, Games Econ. Behav. 102, 433 (2017).

[29] O. Weisel and S. Shalvi, The Collaborative Roots of Corruption, Proc. Natl. Acad. Sci. U.S.A. 112, 10651 (2015).

[30] A. Cassar, Coordination and Cooperation in Local, Random and Small World Networks: Experimental Evidence, Games Econ. Behav. 58, 209 (2007).

[31] O. Kirchkamp and R. Nagel, Naive Learning and Cooperation in Network Experiments, Games Econ. Behav. 58, 269 (2007).

[32] A. Traulsen, D. Semmann, R. D. Sommerfeld, H. J. Krambeck, and M. Milinski, Human Strategy Updating in Evolutionary Games, Proc. Natl. Acad. Sci. U.S.A. 107, 2962 (2010).

[33] J. Grujić, C. Fosco, L. Araujo, J. A. Cuesta, and A. Sánchez, Social Experiments in the Mesoscale: Humans Playing a Spatial Prisoner's Dilemma, PLoS One 5, e13749 (2010).

[34] S. Suri and D. J. Watts, Cooperation and Contagion in WebBased, Networked Public Goods Experiments, PLoS One 6, e16836 (2011).

[35] C. Gracia-Lázaro, A. Ferrer, G. Ruiz, A. Tarancón, J. A. Cuesta, A. Sánchez, and Y. Moreno, Heterogeneous Networks Do Not Promote Cooperation When Humans Play a Prisoner's Dilemma, Proc. Natl. Acad. Sci. U.S.A. 109, 12922 (2012).

[36] J. Grujić, C. Gracia-Lázaro, M. Milinski, D. Semmann, A. Traulsen, J. A. Cuesta, Y. Moreno, and A. Sánchez, A Comparative Analysis of Spatial Prisoner's Dilemma 
Experiments: Conditional Cooperation and Payoff Irrelevance, Sci. Rep. 4, 4615 (2014).

[37] D. G. Rand, M. A. Nowak, J. H. Fowler, and N. A. Christakis, Static Network Structure Can Stabilize Human Cooperation, Proc. Natl. Acad. Sci. U.S.A. 111, 17093 (2014).

[38] A. Mao, L. Dworkin, S. Suri, and D. J. Watts, Resilient Cooperators Stabilize Long-Run Cooperation in the Finitely Repeated Prisoner's Dilemma, Nat. Commun. 8, 13800 (2017).

[39] A. Sánchez, Physics of Human Cooperation: Experimental Evidence and Theoretical Models, J. Stat. Mech. (2018) $\mathrm{P} 024001$.

[40] P. Bak, C. Tang, and K. Wiesenfeld, Self-Organized Criticality: An Explanation of the $1 / f$ Noise, Phys. Rev. Lett. 59, 381 (1987).

[41] P. Bak, C. Tang, and K. Wiesenfeld, Self-Organized Criticality, Phys. Rev. A 38, 364 (1988).

[42] P. Bak and K. Sneppen, Punctuated Equilibrium and Criticality in a Simple Model of Evolution, Phys. Rev. Lett. 71, 4083 (1993).

[43] S. A. Kauffman, The Origins of Order: Self-Organization and Selection in Evolution (Oxford University, New York, 1993).

[44] S. Kauffman, At Home in the Universe: The Search for the Laws of Self-Organization and Complexity (Oxford University, New York, 1996).

[45] P. Bak, How Nature Works: The Science of Self-Organized Criticality (Springer Science \& Business Media, New York, 1996).

[46] R. Dickman, M. A. Muñoz, A. Vespignani, and S. Zapperi, Paths to Self-Organized Criticality, Braz. J. Phys. 30, 27 (2000).
[47] M. A. Munoz, Colloquium: Criticality and Dynamical Scaling in Living Systems, Rev. Mod. Phys. 90, 031001 (2018).

[48] D. Sornette, Critical Phenomena in Natural Sciences: Chaos, Fractals, Self-Organization and Disorder: Concepts and Tools (Springer Science \& Business Media, New York, 2006).

[49] https://github.com/brigan/RiggedEconomies

[50] https://en.wikipedia.org/wiki/Keith_Gill.

[51] Wikipedia, Dai, https://en.wikipedia.org/wiki/Dai_ (cryptocurrency).

[52] Wikipedia, Decentralized Autonomous Organization, en .wikipedia.org/wiki/Decentralized_autonomous_organization.

[53] Q. DuPont, Experiments in Algorithmic Governance: A History and Ethnography of "The DAO," a Failed Decentralized Autonomous Organization, Bitcoin and Beyond 157 (2017), https://doi.org/10.4324/9781315211909-8.

[54] P. Praitheeshan, L. Pan, J. Yu, J. Liu, and R. Doss, Security Analysis Methods on Ethereum Smart Contract Vulnerabilities: A Survey, arXiv:1908.08605.

[55] G. J. Stigler, The Economics of Information, J. Polit. Econ. 69, 213 (1961).

[56] K. J. Arrow, The Economics of Information (Harvard University, Cambridge, MA, 1984), Vol. 4.

[57] K. J. Arrow, The Economics of Information: An Exposition, Empirica 23, 119 (1996).

[58] C. C. Coello, Evolutionary Multi-Objective Optimization: A Historical View of the Field, IEEE Comput. Intell. Mag. 1, 28 (2006).

[59] P. Schuster, Optimization of Multiple Criteria: Pareto Efficiency and Fast Heuristics Should Be More Popular than They Are, Complexity 18, 5 (2012).

[60] L. F. Seoane, Ph.D. dissertation, Universitat Pompeu Fabra, 2016. 